\title{
Six-dimensional beam-beam kick including coupled motion
}

\author{
L.H. A. Leunissen \\ Department of Applied Physics, Delft University of Technology, Delft, The Netherlands \\ F. Schmidt \\ SL-Division, CERN, Geneva, Switzerland \\ G. Ripken \\ Deutsches Elektronen-Synchrotron DESY, Hamburg, Germany \\ (Received 15 March 2000; published 18 December 2000)
}

\begin{abstract}
The six-dimensional beam-beam interaction as developed in 1992 by Hirata, Moshammer, and Ruggiero has been extended to include linear coupled motion and an arbitrary crossing plane. The technique of symplectic mapping in the six-dimensional phase space, called synchrobeam mapping, is applied to investigate the beam-beam kick within a solenoid. A linear beam-beam model including coupling is discussed in detail, also in the framework of a six-dimensional symplectic dispersion formalism.
\end{abstract}

PACS numbers: 41.85.-p, 29.27.-a

\section{INTRODUCTION}

The beam-beam interaction is studied in storage rings, using the formalism developed by Hirata, Moshammer, and Ruggiero [synchrobeam mapping (SBM) and a Lorentz boost transforming the collision with a crossing angle to a head-on collision]. In this approach the strong bunch is split longitudinally into several slices, where each slice is described by an electrostatic potential of the form

$$
\begin{aligned}
U\left(x, y ; \Sigma_{11}, \Sigma_{33}\right)= & -\frac{r_{p}}{\gamma_{0}} \\
& \times \int_{0}^{\infty} \frac{\exp \left(-\frac{x^{2}}{2 \Sigma_{11}+u}-\frac{y^{2}}{2 \Sigma_{33}+u}\right)}{\sqrt{2 \Sigma_{11}+u} \sqrt{2 \Sigma_{33}+u}} d u .
\end{aligned}
$$

Here $r_{p}$ is the classical particle radius, $\gamma_{0}$ is the Lorentz factor of the test particle, and $\underline{\Sigma}$ is the $6 \times 6$ phase-space envelope matrix of the strong bunch defined by

$$
\Sigma_{i j} \equiv\left\langle X_{i} X_{j}\right\rangle-\left\langle X_{i}\right\rangle\left\langle X_{j}\right\rangle, \quad i, j=1, \ldots, 6,
$$

where the lowercase $x, y$ and the uppercase $X, Y$ stand for the transverse coordinates of the test particle and the strong bunch with $\vec{X}=\left(X, P_{X} ; Y, P_{Y} ; Z, P_{Z}\right)^{T}$, respectively. In addition, a new technique of symplectic mapping in the six-dimensional phase space, called synchrobeam mapping, has been introduced by these authors in Ref. [1]. It allows one to include the bunch length effect at the collision point and the energy variation caused by the electric field of the opposite bunch. This mapping is formulated only for head-on collision, but Hirata has shown that a crossing angle can be eliminated by a Lorentz boost [2].
Equation (1.1) is valid for the case of uncoupled motion. The aim of this report is to extend the formalism to include six-dimensional linear coupling.

This paper is organized as follows: In Sec. II the beam-beam kick is studied in the most general form. The tilted cross section induced by coupling, which is needed in Sec. II, is calculated in Appendix A. In Appendix B we describe methods to construct SBM solutions for a solenoid field which allow us to investigate the beam-beam kick within a solenoid. The SBM solution is obtained by the use of a generating function, Lie series, or by an integration method. A linear model of the beam-beam kick including coupling is studied in Appendices C and D, concerning the derivation of the linear beam-beam matrix, the tune shift caused by a beam-beam kick and a linear sixdimensional dispersion formalism including the beambeam interaction. Last, a summary of the results is presented in Sec. III. The 6D beam-beam formalism has been incorporated in the tracking programs MAD [3] and SIXTRACK [4].

\section{BEAM-BEAM KICK FOR COUPLED MOTION}

\section{A. The electromagnetic field due to a tilted bunch}

The generalization of the analysis in Refs. [1,2] by including coupling and a tilted strong bunch (caused by coupling) can be achieved in a straightforward manner by describing the particle motion in the framework of the fully coupled six-dimensional formalism and by replacing the electric potential $U$ of Eq. (1.1) for an untilted bunch by a new potential

$$
\hat{U}\left(x, y ; \hat{\Sigma}_{11}, \hat{\Sigma}_{33} ; \theta\right) \equiv U\left(\hat{x}, \hat{y} ; \hat{\Sigma}_{11}, \hat{\Sigma}_{33}\right)=-\frac{r_{p}}{\gamma_{0}} \int_{0}^{\infty} \frac{\exp \left(-\frac{\hat{x}^{2}}{2 \hat{\Sigma}_{11}+u}-\frac{\hat{y}^{2}}{2 \hat{\Sigma}_{33}+u}\right)}{\sqrt{2 \hat{\Sigma}_{11}+u} \sqrt{2 \hat{\Sigma}_{33}+u}} d u,
$$


where the symbol ^ denotes quantities in the coupled frame of reference. The coupling has to be considered for the test particle as well as for the strong bunch.

\section{Test particle}

The potential (2.1) is obtained from (1.1) by introducing a rotated coordinate system of the test particle (for details see Appendix A)

$$
\hat{x}=x \cos \theta+y \sin \theta, \quad \hat{y}=-x \sin \theta+y \cos \theta,
$$

where $\theta$ denotes the coupling angle of the strong bunch given by ${ }^{1}$

$$
\begin{aligned}
\sin 2 \theta & =-\operatorname{sgn}\left(\Sigma_{11}-\Sigma_{33}\right) \frac{2 \Sigma_{13}}{\sqrt{\left[\Sigma_{11}-\Sigma_{33}\right]^{2}+4 \Sigma_{13}^{2}}} \\
\cos 2 \theta & =\operatorname{sgn}\left(\Sigma_{11}-\Sigma_{33}\right) \frac{\left(\Sigma_{11}-\Sigma_{33}\right)}{\sqrt{\left[\Sigma_{11}-\Sigma_{33}\right]^{2}+4 \Sigma_{13}^{2}}} \\
& \Longrightarrow \tan 2 \theta=-\frac{2 \Sigma_{13}}{\Sigma_{11}-\Sigma_{33}},
\end{aligned}
$$

or

$$
\begin{aligned}
& \hat{\Sigma}_{11} \equiv\left\langle\hat{X}^{2}\right\rangle=\frac{1}{2}\left\{\left[\Sigma_{11}+\Sigma_{33}\right]+\operatorname{sgn}\left(\Sigma_{11}-\Sigma_{33}\right) \sqrt{\left[\Sigma_{11}-\Sigma_{33}\right]^{2}+4 \Sigma_{13}^{2}}\right\}, \\
& \hat{\Sigma}_{33} \equiv\left\langle\hat{Y}^{2}\right\rangle=\frac{1}{2}\left\{\left[\Sigma_{11}+\Sigma_{33}\right]-\operatorname{sgn}\left(\Sigma_{11}-\Sigma_{33}\right) \sqrt{\left[\Sigma_{11}-\Sigma_{33}\right]^{2}+4 \Sigma_{13}^{2}}\right\} .
\end{aligned}
$$

These elements are a function of the eigenvectors

$$
\begin{aligned}
& \Sigma_{11} \equiv\left\langle X^{2}\right\rangle=\sum_{k=I, I I, I I I} 2 J_{k} v_{k 1} v_{k 1}^{*}, \\
& \Sigma_{33} \equiv\left\langle Y^{2}\right\rangle=\sum_{k=I, I I, I I I} 2 J_{k} v_{k 3} v_{k 3}^{*}, \\
& \Sigma_{13} \equiv\langle X Y\rangle=\sum_{k=I, I I, I I I} J_{k}\left[v_{k 1} v_{k 3}^{*}+v_{k 1}^{*} v_{k 3}\right] .
\end{aligned}
$$

Note that

$$
E_{1}=\sqrt{\hat{\Sigma}_{11}}, \quad E_{2}=\sqrt{\hat{\Sigma}_{33}}
$$

are the principal axes of the elliptical cross section

$$
\frac{\hat{X}^{2}}{E_{1}}+\frac{\hat{Y}^{2}}{E_{2}}=1
$$

in the $(\hat{X}-\hat{Y})$ plane.

\footnotetext{
${ }^{1}$ We have chosen $\theta=-\theta_{p}$ defined in Appendix A since the strong bunch rotates in the opposite direction of the test particle. In a double-ring machine, such as the Large Hadron Collider, this is not necessarily true.
}

Conversely to Ref. [2], the crossing angle $2 \phi$ can be chosen in an arbitrary crossing plane $(\tilde{x}-s)$, defined by an angle $\alpha$ (see Fig. 1). We can write the components of the strong bunch in a Cartesian coordinate system $\left(X, Y, Z ; P_{x}, P_{y}, P_{y}\right)$ defined for the laboratory frame and oriented according to the ideal orbit of the test particle as

$$
\begin{gathered}
P_{x}=P \sin 2 \phi \cos \alpha, \quad P_{y}=P \sin 2 \phi \sin \alpha, \\
P_{z}=-P \cos 2 \phi,
\end{gathered}
$$

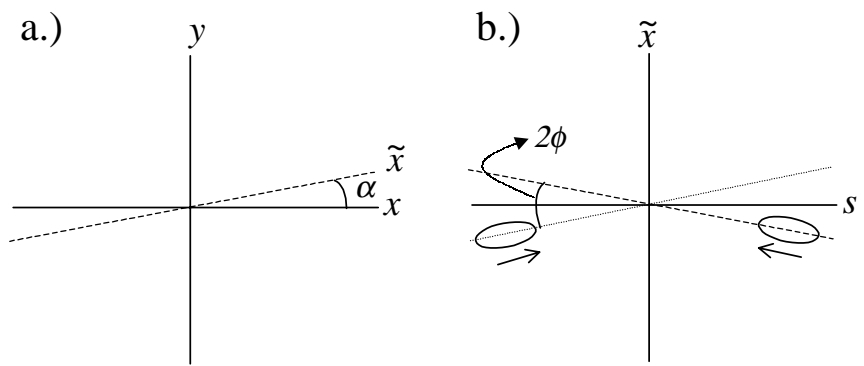

FIG. 1. (a) defines the crossing plane angle $\alpha$ in the $(x-y)$ plane and (b) depicts the total crossing angle $2 \phi$ in the $(\tilde{x}-s)$ plane. 
with $P$ the momentum of the bunch.

\section{B. Lorentz boost}

The Lorentz boost as described by Hirata consists of a transformation of Cartesian to accelerator coordinates and a Lorentz boost which makes the collision between the bunches head-on. This is necessary because the 6D beambeam interaction is only described for a head-on collision. In addition, we include the crossing plane angle $\alpha$ in our formalism.

\section{Transformation from Cartesian to accelerator coordinates}

The following relations of Ref. [2] remain valid:

$$
\left(\begin{array}{c}
c t \\
x_{C} \\
z_{C} \\
y_{C}
\end{array}\right)=\underline{A}\left(\begin{array}{c}
z(s) \\
x(s) \\
s \\
y(s)
\end{array}\right)
$$

where

$$
\underline{A}=\underline{A}^{-1}=\left(\begin{array}{cccc}
-1 & 0 & 1 & 0 \\
0 & 1 & 0 & 0 \\
0 & 0 & 1 & 0 \\
0 & 0 & 0 & 1
\end{array}\right)
$$

and

$$
\left(\begin{array}{c}
E / c-p_{0} \\
p_{x C} \\
p_{z C}-p_{0} \\
p_{y C}
\end{array}\right)=p_{0} \underline{B}\left(\begin{array}{c}
p_{z} \\
p_{x} \\
h \\
p_{y}
\end{array}\right)
$$

with

$$
\left(\begin{array}{cccc}
1 / \cos \phi & -\cos \alpha \sin \phi & -\tan \phi \sin \phi & -\sin \alpha \sin \phi \\
-\cos \alpha \tan \phi & 1 & \cos \alpha \tan \phi & 0 \\
0 & -\cos \alpha \sin \phi & \cos \phi & -\sin \alpha \sin \phi \\
-\sin \alpha \tan \phi & 0 & \sin \alpha \tan \phi & 1
\end{array}\right)
$$

In order to interpret $\underline{L}$ in Eq. (2.19) we may introduce a new coordinate system

$$
\left(\begin{array}{l}
\tilde{x} \\
\tilde{y}
\end{array}\right)=\left(\begin{array}{cc}
\cos \alpha & \sin \alpha \\
-\sin \alpha & \cos \alpha
\end{array}\right)\left(\begin{array}{l}
x \\
y
\end{array}\right)
$$

corresponding to $\underline{R}$. Then the crossing plane is identical to the $(\tilde{x}-s)$ plane (see Fig. 1). Since $\underline{L}_{0}$ can be written in the form

$$
\underline{L}_{0}=\underbrace{\left(\begin{array}{cccc}
1 / \cos \phi & -\tan \phi & 0 & 0 \\
-\tan \phi & 1 / \cos \phi & 0 & 0 \\
0 & 0 & 1 & 0 \\
0 & 0 & 0 & 1
\end{array}\right)}_{\begin{array}{c}
\text { Boost in the direction of } \\
\text { the rotated } \tilde{x} \text { axis }
\end{array}} \underbrace{\left(\begin{array}{cccc}
1 & 0 & 0 & 0 \\
0 & \cos \phi & \sin \phi & 0 \\
0 & -\sin \phi & \cos \phi & 0 \\
0 & 0 & 0 & 1
\end{array}\right)}_{\begin{array}{c}
\text { Rotation in the } \\
(s-\tilde{x}) \text { plane }
\end{array}}
$$


we define a second coordinate system

$$
\left(\begin{array}{l}
\bar{x} \\
\bar{s}
\end{array}\right)=\left(\begin{array}{cc}
\cos \phi & \sin \phi \\
-\sin \phi & \cos \phi
\end{array}\right)\left(\begin{array}{l}
\tilde{x} \\
s
\end{array}\right) .
$$

Then we are left with a boost in the direction of $\bar{x}$ representing the rotated $\tilde{x}$ axis,

$$
\left(\begin{array}{c}
t^{*} \\
\bar{x}^{*}
\end{array}\right)=\left(\begin{array}{cc}
1 / \cos \phi & -\tan \phi \\
-\tan \phi & 1 / \cos \phi
\end{array}\right)\left(\begin{array}{c}
t \\
\bar{x}
\end{array}\right) .
$$

\section{Full Lorentz boost}

As a result of the Lorentz boost we have

$$
\begin{aligned}
\left(\begin{array}{c}
c T^{*} \\
X^{*} \\
Z^{*} \\
Y^{*}
\end{array}\right) & =\underline{L}\left(\begin{array}{c}
c T \\
X \\
Z \\
Y
\end{array}\right), \\
\left(\begin{array}{c}
E^{*} / c \\
P_{X}^{*} \\
P_{Z}^{*} \\
P_{Y}^{*}
\end{array}\right) & =\underline{L}\left(\begin{array}{c}
E / c \\
P_{X} \\
P_{Z} \\
P_{Y}
\end{array}\right) .
\end{aligned}
$$

Inserting Eq. (2.12) into Eq. (2.27), we get for the transformed momentum of the strong bunch $(E / c=P) P_{x}^{*}=$ $0, P_{y}^{*}=0$ since

$$
\begin{aligned}
P_{x}^{*}= & -P(\cos \alpha \tan \phi-\sin 2 \phi \cos \alpha \\
& \quad+\cos \alpha \tan \phi \cos 2 \phi) \\
= & -P \cos \alpha \tan \phi\left(1-2 \cos ^{2} \phi+\cos ^{2} \phi-\sin ^{2} \phi\right) \\
= & 0, \\
P_{y}^{*}= & -P(\sin \alpha \tan \phi+\sin \alpha \tan \phi \cos 2 \phi \\
& \quad-\quad-\sin 2 \phi \sin \alpha) \\
= & 0 .
\end{aligned}
$$

For the test particle $p_{x}=p_{y}=0, E=c p_{0}$ is transformed into $p_{x}^{*}=p_{y}^{*}=0$ and $E^{*}=c p_{0}^{*}=c p_{0} \cos \phi$, i.e., the collision is indeed head-on.

Using Eq. (2.21), the full Lorentz transformation is therefore a transformation from the accelerator coordinates to Cartesian coordinates, the Lorentz transformation, and again a backwards transformation to the accelerator coordinates

$$
\vec{x}(0) \rightarrow \vec{x}^{*}\left(s^{*}\right)
$$

leading to

$$
\left(\begin{array}{c}
z^{*}\left(s^{*}\right) \\
x^{*}\left(s^{*}\right) \\
s^{*} \\
y^{*}\left(s^{*}\right)
\end{array}\right)=\underline{A}^{-1} \underline{L A}\left(\begin{array}{c}
z(0) \\
x(0) \\
0 \\
y(0)
\end{array}\right)=\left(\begin{array}{cccc}
1 / \cos \phi & 0 & 0 & 0 \\
\cos \alpha \tan \phi & 1 & 0 & 0 \\
0 & -\cos \alpha \sin \phi & \cos \phi & -\sin \alpha \sin \phi \\
\sin \alpha \tan \phi & 0 & 0 & 1
\end{array}\right)\left(\begin{array}{c}
z(0) \\
x(0) \\
0 \\
y(0)
\end{array}\right)
$$

and

$$
\left(\begin{array}{c}
p_{z}^{*}\left(s^{*}\right) \\
p_{x}^{*}\left(s^{*}\right) \\
h^{*} \\
p_{y}^{*}\left(s^{*}\right)
\end{array}\right)=\frac{p_{0}}{p_{0}^{*}} \underline{B}^{-1} \underline{L B}\left(\begin{array}{c}
p_{z}(0) \\
p_{x}(0) \\
h \\
p_{y}(0)
\end{array}\right)=\left(\begin{array}{cccc}
1 & -\cos \alpha \tan \phi & \tan ^{2} \phi & -\sin \alpha \tan \phi \\
0 & 1 / \cos \phi & -\cos \alpha \frac{\tan \phi}{\cos \phi} & 0 \\
0 & 0 & 1 / \cos ^{2} \phi & 0 \\
0 & 0 & -\sin \alpha \frac{\tan \phi}{\cos \phi} & 1 / \cos \phi
\end{array}\right)\left(\begin{array}{c}
p_{z}(0) \\
p_{x}(0) \\
h \\
p_{y}(0)
\end{array}\right) .
$$

From Eq. (2.30) we have

$$
s^{*}=-x(0) \cos \alpha \sin \phi-y(0) \sin \alpha \sin \phi,
$$

so that, in general, $s=0$ is not necessarily transformed to $s^{*}=0$. Since we need a transformation from $\vec{x}(0)$ to $\vec{x}^{*}\left(0^{*}\right)$, an additional transformation

$$
\vec{x}^{*}\left(s^{*}\right) \rightarrow \vec{x}^{*}\left(0^{*}\right)
$$

has to be performed.

Following Ref. [2], the transformation (2.33) can be written as a first-order Taylor expansion

$$
\begin{aligned}
w_{i}^{*}\left(0^{*}\right) & =w_{i}^{*}\left(s^{*}\right)-\frac{d w_{i}^{*}\left(0^{*}\right)}{d s^{*}} s^{*}=w_{i}^{*}\left(s^{*}\right)-h_{i}^{*} s^{*} \\
& =w_{i}^{*}\left(s^{*}\right)+h_{i}^{*} \sin \phi[x(0) \cos \alpha+y(0) \sin \alpha],
\end{aligned}
$$

with

$$
\begin{aligned}
& w_{i} \equiv(x, y, z), \\
& h_{i}^{*}=\frac{\partial}{\partial p_{i}^{*}} h^{*}\left(p_{x}^{*}, p_{y}^{*}, p_{z}^{*} ; p_{0}^{*}\right) .
\end{aligned}
$$

Furthermore, we obtain from (2.31) and the Hamiltonian (2.17)

$$
\begin{aligned}
h^{*}\left(p_{x}^{*}, p_{y}^{*}, p_{z}^{*} ; p_{0}^{*}\right) & =\frac{h\left(p_{x}, p_{y}, p_{z} ; p_{0}\right)}{\cos ^{2} \phi} \\
& =h\left(p_{x}^{*}, p_{y}^{*}, p_{z}^{*} ; p_{0}^{*}\right)
\end{aligned}
$$

Combining the transformations (2.30), (2.31), and (2.34), we finally obtain the equations 


$$
\begin{aligned}
& x^{*}=z \cos \alpha \tan \phi+x+h_{x}^{*}[x \cos \alpha \sin \phi+y \sin \alpha \sin \phi]=z \cos \alpha \tan \phi+x\left[1+h_{x}^{*} \cos \alpha \sin \phi\right]+y h_{x}^{*} \sin \alpha \sin \phi, \\
& y^{*}=z \sin \alpha \tan \phi+y+h_{y}^{*}[x \cos \alpha \sin \phi+y \sin \alpha \sin \phi]=z \sin \alpha \tan \phi+y\left[1+h_{y}^{*} \sin \alpha \sin \phi\right]+x h_{y}^{*} \cos \alpha \sin \phi,
\end{aligned}
$$

with

$$
\begin{aligned}
\tilde{x}=x, \quad \tilde{y}=y, \quad \tilde{z}=z, \\
\tilde{p}_{x}=\frac{p_{x}}{\cos \phi}, \quad \tilde{p}_{y}=\frac{p_{y}}{\cos \phi}, \quad \tilde{p}_{z}=\frac{p_{z}}{\cos \phi}
\end{aligned}
$$

and a canonical transformation

$$
\left(\tilde{x}, \tilde{y}, \tilde{z} ; \tilde{p}_{x}, \tilde{p}_{y}, \tilde{p}_{z}\right) \rightarrow\left(x^{*}, y^{*}, z^{*} ; p_{x}^{*}, p_{y}^{*}, p_{z}^{*}\right)
$$

resulting from the generating function
Thus $\mathcal{L}$ is only quasisymplectic; the Jacobian of this transformation is $1 / \cos ^{3} \phi$. This lack of symplecticity is restored in the backwards transformation $\mathcal{L}^{-1}$ after having applied the beam-beam force.

\section{Beam-beam force}

We approximate the strong bunch by a number of slices. Each slice is represented by its $Z^{*}\left(0^{*}\right)$ coordinate, which shall be denoted by $Z^{\dagger}$. Taking into account only terms linear with respect to dynamical variables in $\mathcal{L}$, the first and second momenta of the particle distribution at the locations of the slices are given by

$$
\begin{gathered}
X^{\dagger}=Z^{\dagger} \cos \alpha \sin \phi, \quad P_{X}^{\dagger}=0, \quad Y^{\dagger}=Z^{\dagger} \sin \alpha \sin \phi, \quad P_{Y}^{\dagger}=0, \quad P_{Z}^{\dagger}=0, \\
\Sigma_{11}^{\dagger}=\Sigma_{11}, \quad \Sigma_{22}^{\dagger}=\frac{1}{\cos ^{2} \phi} \Sigma_{22}, \quad \Sigma_{33}^{\dagger}=\Sigma_{33}, \quad \Sigma_{44}^{\dagger}=\frac{1}{\cos ^{2} \phi} \Sigma_{44}, \quad \Sigma_{12}^{\dagger}=\frac{1}{\cos \phi} \Sigma_{12}, \\
\Sigma_{13}^{\dagger}=\Sigma_{13}, \quad \Sigma_{14}^{\dagger}=\frac{1}{\cos \phi} \Sigma_{14}, \quad \Sigma_{23}^{\dagger}=\frac{1}{\cos \phi} \Sigma_{23}, \quad \Sigma_{24}^{\dagger}=\frac{1}{\cos ^{2} \phi} \Sigma_{24}, \quad \Sigma_{34}^{\dagger}=\frac{1}{\cos \phi} \Sigma_{34} .
\end{gathered}
$$

Inserting Eq. (2.42) into Eqs. (2.3) and (2.8) one obtains

$$
\theta^{\dagger}=\theta, \quad \hat{\Sigma}_{11}^{\dagger}=\hat{\Sigma}_{11}, \quad \hat{\Sigma}_{33}^{\dagger}=\hat{\Sigma}_{33}, \quad \hat{\Sigma}_{13}^{\dagger}=\hat{\Sigma}_{13}
$$

i.e., the cross section of the strong bunch remains unchanged.

$$
\begin{aligned}
& \hat{\Sigma}_{11}^{\dagger}(S)=\frac{1}{2}\left\{\left[\Sigma_{11}^{\dagger}(S)+\Sigma_{33}^{\dagger}(S)\right]+\operatorname{sgn}\left[\Sigma_{11}^{\dagger}(S)-\Sigma_{33}^{\dagger}(S)\right] \sqrt{\left[\Sigma_{11}^{\dagger}(S)-\Sigma_{33}^{\dagger}(S)\right]^{2}+4 \Sigma_{13}^{\dagger}(S)^{2}}\right\}, \\
& \hat{\Sigma}_{33}^{\dagger}(S)=\frac{1}{2}\left\{\left[\Sigma_{11}^{\dagger}(S)+\Sigma_{33}^{\dagger}(S)\right]-\operatorname{sgn}\left[\Sigma_{11}^{\dagger}(S)-\Sigma_{33}^{\dagger}(S)\right] \sqrt{\left[\Sigma_{11}^{\dagger}(S)-\Sigma_{33}^{\dagger}(S)\right]^{2}+4 \Sigma_{13}^{\dagger}(S)^{2}}\right\},
\end{aligned}
$$

i.e., we obtain the same result for a slice and the whole bunch, respectively. In a drift space (e.g., the horizontal plane),

$$
X(S)=X(0)+P_{X}(0) S, \quad P_{X}(S)=P_{X}(0),
$$


we have

$$
\begin{aligned}
& \Sigma_{11}^{\dagger}(S)=\Sigma_{11}^{\dagger}(0)+2 \Sigma_{12}^{\dagger}(0) S+\Sigma_{22}^{\dagger}(0) S^{2}=\Sigma_{11}(0)+2 \Sigma_{12}(0) \varphi+\Sigma_{22}(0) \varphi^{2} \equiv \Sigma_{11}(\varphi) \\
& \Sigma_{33}^{\dagger}(S)=\Sigma_{33}^{\dagger}(0)+2 \Sigma_{34}^{\dagger}(0) S+\Sigma_{44}^{\dagger}(0) S^{2}=\Sigma_{33}(0)+2 \Sigma_{34}(0) \varphi+\Sigma_{44}(0) \varphi^{2} \equiv \Sigma_{33}(\varphi) \\
& \Sigma_{13}^{\dagger}(S)=\Sigma_{13}^{\dagger}(0)+\left[\Sigma_{14}^{\dagger}(0)+\Sigma_{23}^{\dagger}(0)\right] S+\Sigma_{24}^{\dagger}(0) S^{2}=\Sigma_{13}(0)+\left[\Sigma_{14}(0)+\Sigma_{23}(0)\right] \varphi+\Sigma_{24}(0) \varphi^{2} \equiv \Sigma_{13}(\varphi)
\end{aligned}
$$

where $\varphi=\frac{S}{\cos \phi}$. Thus,

$$
\begin{gathered}
\hat{\Sigma}_{11}^{\dagger}(S)=\hat{\Sigma}_{11}(\varphi), \quad \hat{\Sigma}_{33}^{\dagger}(S)=\hat{\Sigma}_{33}(\varphi) \\
\theta^{\dagger}(S)=\theta(\varphi)
\end{gathered}
$$

with $\hat{\Sigma}_{11}, \hat{\Sigma}_{33}$, and $\theta$ given by (2.3) and (2.8).

The real collision between the test particle and the slice takes place at $S$; see Eq. (2.44). To calculate the beambeam interaction, three subsequent transformations have to be performed. First, the test particle at the IP is brought to the CP by a drift. Then the beam-beam interaction is applied and finally the position of the test particle is brought back to the IP. This set of transformations is called the SBM. ${ }^{2}$

It is convenient to introduce a new set of canonical variables at the collision point

$$
\begin{aligned}
& \bar{x}^{*}=x^{*}+S p_{x}^{*}-X^{\dagger}\left(Z^{\dagger}\right), \\
& \bar{y}^{*}=y^{*}+S p_{y}^{*}-Y^{\dagger}\left(Z^{\dagger}\right), \\
& \bar{z}^{*}=z^{*},
\end{aligned}
$$

and

$$
\begin{gathered}
\bar{p}_{x}^{*}=p_{x}^{*}, \quad \bar{p}_{y}^{*}=p_{y}^{*}, \\
\bar{p}_{z}^{*}=p_{z}^{*}-\frac{\left(p_{x}^{*}\right)^{2}+\left(p_{y}^{*}\right)^{2}}{4} .
\end{gathered}
$$

Here we have assumed a drift space between the IP and the CP. In these new variables, the SBM transformation can be written as a concatenation of three symplectic transformations,

$$
\exp (-: D:) \exp \left(: \mathcal{H}_{b b}:\right) \exp (: D:)
$$

\footnotetext{
${ }^{2}$ The SBM as described in detail in Ref. [1] can be represented by a Hamiltonian $\mathcal{H}=\mathcal{H}_{b b}\left(\vec{x}^{*}\right) \delta\left(s^{*}\right)$ with $\mathcal{H}_{b b}$ defined implicitly by $\exp \left(: \mathcal{H}_{b b}:\right)=\prod_{Z^{\dagger}} \exp \left(: n^{*} U\left(\hat{x}^{*}, \hat{y}^{*} ; \hat{\Sigma}_{11}, \hat{\Sigma}_{33}\right):\right)$ describing the interaction of a test particle in the weak bunch with a slice of the strong bunch represented by $Z^{\dagger}$.
}

where

$$
D(S)=\frac{\left(p_{x}^{*}\right)^{2}+\left(p_{y}^{*}\right)^{2}}{2} S
$$

Note that there is an additional transformation, i.e., a shift by $X^{\dagger}\left(Z^{\dagger}\right)$ and $Y^{\dagger}\left(Z^{\dagger}\right)$; see Eq. (2.49). In Appendix B, $D(S)$ is calculated in the presence of a solenoid field.

The particle-slice interaction at the CP finally leads to

$$
\left(\bar{x}^{*}, \bar{y}^{*}, \bar{z}^{*}\right) \rightarrow\left(\bar{x}^{*}, \bar{y}^{*}, \bar{z}^{*}\right)
$$

and

$$
\begin{gathered}
\bar{p}_{x}^{*} \rightarrow \bar{p}_{x}^{*}-n^{*} F_{x}^{*}, \quad \bar{p}_{y}^{*} \rightarrow \bar{p}_{y}^{*}-n^{*} F_{y}^{*}, \\
\bar{p}_{z}^{*} \rightarrow \bar{p}_{z}^{*}-n^{*} F_{z}^{*},
\end{gathered}
$$

where $n^{*}$ is the number of particles in the slice, i.e., the total number $N^{*}$ divided by the number of slices, and

$$
\begin{aligned}
F_{x}^{*} & =\frac{\partial}{\partial \bar{x}^{*}} \hat{U}\left(\bar{x}^{*}, \bar{y}^{*} ; \hat{\Sigma}_{11}(\varphi), \hat{\Sigma}_{33}(\varphi) ; \theta(\varphi)\right), \\
F_{y}^{*} & =\frac{\partial}{\partial \bar{y}^{*}} \hat{U}\left(\bar{x}^{*}, \bar{y}^{*} ; \hat{\Sigma}_{11}(\varphi), \hat{\Sigma}_{33}(\varphi) ; \theta(\varphi)\right), \\
F_{z}^{*} & =\frac{\partial}{\partial \bar{z}^{*}} \hat{U}\left(\bar{x}^{*}, \bar{y}^{*} ; \hat{\Sigma}_{11}(\varphi), \hat{\Sigma}_{33}(\varphi) ; \theta(\varphi)\right) \\
& =\frac{1}{2} \frac{\partial}{\partial S} \hat{U}\left(\bar{x}^{*}, \bar{y}^{*} ; \hat{\Sigma}_{11}(\varphi), \hat{\Sigma}_{33}(\varphi) ; \theta(\varphi)\right),
\end{aligned}
$$

with $\hat{U}$ given by Eq. (2.1).

Introducing the variables

$$
\underline{x}^{*}=w_{1} \bar{x}^{*}+w_{2} \bar{y}^{*}, \quad \underline{y}^{*}=-w_{2} \bar{x}^{*}+w_{1} \bar{y}^{*}
$$

[see Eq. (2.2)] with

$$
w_{1}=\cos \theta, \quad w_{2}=\sin \theta,
$$

we can also write 


$$
\begin{aligned}
F_{x}^{*} & =\frac{\partial}{\partial \bar{x}^{*}} U\left(\underline{x}^{*}, \underline{y}^{*} ; \hat{\Sigma}_{11}(\varphi), \hat{\Sigma}_{33}(\varphi)\right)=w_{1}(\varphi) \frac{\partial}{\partial \underline{x}^{*}} U\left(\underline{x}^{*}, \underline{y}^{*} ; \hat{\Sigma}_{11}(\varphi), \hat{\Sigma}_{33}(\varphi)\right)-w_{2}(\varphi) \frac{\partial}{\partial \underline{y}^{*}} U\left(\underline{x}^{*}, \underline{y}^{*} ; \hat{\Sigma}_{11}(\varphi), \hat{\Sigma}_{33}(\varphi)\right), \\
F_{y}^{*} & =\frac{\partial}{\partial \bar{y}^{*}} U\left(\underline{x}^{*}, \underline{y}^{*} ; \hat{\Sigma}_{11}(\varphi), \hat{\Sigma}_{33}(\varphi)\right) \\
& =w_{2}(\varphi) \frac{\partial}{\partial \underline{x}^{*}} U\left(\underline{x}^{*}, \underline{y}^{*} ; \hat{\Sigma}_{11}(\varphi), \hat{\Sigma}_{33}(\varphi)\right)+w_{1}(\varphi) \frac{\partial}{\partial \underline{y}^{*}} U\left(\underline{x}^{*}, \underline{y}^{*} ; \hat{\Sigma}_{11}(\varphi), \hat{\Sigma}_{33}(\varphi)\right),
\end{aligned}
$$$$
F_{z}^{*}=\frac{1}{2} \frac{\partial}{\partial S} U\left(\underline{x}^{*}, \underline{y}^{*} ; \hat{\Sigma}_{11}(\varphi), \hat{\Sigma}_{33}(\varphi)\right)
$$$$
=\frac{\partial U}{\partial \underline{x}^{*}}\left[w_{1}^{\prime}(\varphi) \bar{x}^{*}+w_{2}^{\prime}(\varphi) \bar{y}^{*}\right] \frac{1}{2 \cos \phi}+\frac{\partial U}{\partial \underline{y}^{*}}\left[-w_{2}^{\prime}(\varphi) \bar{x}^{*}+w_{1}^{\prime}(\varphi) \bar{y}^{*}\right] \frac{1}{2 \cos \phi}
$$$$
+\frac{\partial U}{\partial \hat{\Sigma}_{11}} \hat{\Sigma}_{11}^{\prime}(\varphi) \frac{1}{2 \cos \phi}+\frac{\partial U}{\partial \hat{\Sigma}_{33}} \hat{\Sigma}_{33}^{\prime}(\varphi) \frac{1}{2 \cos \phi},
$$

with $U$ defined in Eq. (1.1) and the symbol ' indicating a differentiation with respect to $\varphi$.

Expressions for the terms $\partial U / \partial \underline{x}^{*}, \partial U / \partial \underline{y}^{*}, \partial U / \partial \hat{\Sigma}_{11}$, and $\partial U / \partial \hat{\Sigma}_{33}$ appearing in Eq. (2.58) can be found in Ref. [1] [see Eqs. (21), (22), (86), and (87)] for a triGaussian distribution.

The terms $\hat{\Sigma}_{11}^{\prime}(\varphi)$ and $\hat{\Sigma}_{33}^{\prime}(\varphi)$ may be obtained by using Eqs. (2.8) and (2.9) and by taking into account that the eigenvectors $\vec{v}_{k}(s)(k=I, I I, I I I)$ obey the equations of motion. For a drift space ${ }^{3}$ they read

$$
\begin{gathered}
\frac{d}{d s} \boldsymbol{v}_{k 1}=\boldsymbol{v}_{k 2}, \quad \frac{d}{d s} \boldsymbol{v}_{k 3}=\boldsymbol{v}_{k 4}, \\
\frac{d}{d s} \boldsymbol{v}_{k 2}=\frac{d}{d s} \boldsymbol{v}_{k 4}=0 .
\end{gathered}
$$

Using Eq. (2.3) we obtain

$$
\begin{aligned}
& \hat{\Sigma}_{11}^{\prime}(\varphi)=C_{0}+C_{1} \cos [2 \theta(\varphi)]-C_{2} \sin [2 \theta(\varphi)], \\
& \hat{\Sigma}_{33}^{\prime}(\varphi)=C_{0}-C_{1} \cos [2 \theta(\varphi)]+C_{2} \sin [2 \theta(\varphi)],
\end{aligned}
$$

with

$$
\begin{aligned}
& C_{0}=\Sigma_{12}(0)+\Sigma_{34}(0)+\left(\Sigma_{22}(0)+\Sigma_{44}(0)\right) \varphi \\
& C_{1}=\Sigma_{12}(0)-\Sigma_{34}(0)+\left(\Sigma_{22}(0)-\Sigma_{44}(0)\right) \varphi \\
& C_{2}=\Sigma_{14}(0)+\Sigma_{23}(0)+2 \Sigma_{24}(0) \varphi
\end{aligned}
$$

The quantities $w_{1}$ and $w_{2}$ are determined by Eqs. (2.3) and (2.57). Last, in order to calculate $w_{1}^{\prime}(s)$ and $w_{2}^{\prime}(s)$ we use the relations

\footnotetext{
${ }^{3}$ In this case Eq. (2.47) can also serve to calculate the terms $\Sigma_{11}^{\prime}(\varphi), \Sigma_{33}^{\prime}(\varphi)$, and $\Sigma_{13}^{\prime}(\varphi)$ appearing in $\hat{\Sigma}_{11}^{\prime}$ and $\hat{\Sigma}_{33}^{\prime}$.
}

$$
\begin{gathered}
\cos 2 \theta=\cos ^{2} \theta-\sin ^{2} \theta=2 \cos ^{2} \theta-1=1-2 \sin ^{2} \theta \\
\Longrightarrow\left\{\begin{array}{l}
w_{1}^{\prime}(s) \equiv \frac{d}{d s} \cos \theta=\frac{1}{4 \cos \theta} \frac{d}{d s} \cos 2 \theta, \\
w_{2}^{\prime}(s) \equiv \frac{d}{d s} \sin \theta=-\frac{1}{4 \sin \theta} \frac{d}{d s} \cos 2 \theta
\end{array}\right.
\end{gathered}
$$

where the trigonometric functions are taken from Eqs. (2.3) and (2.4).

Going back to the original coordinates, the explicit form for the complete SBM is given by

$$
\begin{aligned}
x_{\text {new }}^{*}= & x^{*}+S n^{*} F_{x}^{*}, \quad p_{x, \text { new }}^{*}=p_{x}^{*}-n^{*} F_{x}^{*}, \\
y_{\text {new }}^{*}= & y^{*}+S n^{*} F_{y}^{*}, \quad p_{y, \text { new }}^{*}=p_{y}^{*}-n^{*} F_{y}^{*}, \\
z_{\text {new }}^{*}= & z^{*}, \\
p_{z \text {,new }}^{*}= & p_{z}^{*}-n^{*} F_{z}^{*} \\
& -\frac{1}{2}\left[n^{*} F_{x}^{*}\left(p_{x}^{*}-\frac{n^{*} F_{x}^{*}}{2}\right)\right. \\
& \left.\quad+n^{*} F_{y}^{*}\left(p_{y}^{*}-\frac{n^{*} F_{y}^{*}}{2}\right)\right] .
\end{aligned}
$$

\section{SUMMARY}

We have studied the beam-beam interaction for coupled motion in the framework of the weak-strong formalism taking into account a tilted cross section of the strong beam induced by linear coupling. This coupling has been included in the 6D beam-beam formalism of Hirata, Moshammer, and Ruggiero.

The extended formalism also allows for an arbitrary crossing plane. Furthermore, a SBM solution for solenoid fields is derived which allows one to investigate the beam-beam kick within a solenoid.

A linear model of the beam-beam kick, due to a tilted cross section of the strong bunch, is investigated in detail in Appendices $\mathrm{C}$ and $\mathrm{D}$ (beam-beam matrix and dispersion formalism including beam-beam interaction). 
The equations derived in this report have been incorporated into the tracking codes MAD and SIXTRACK.

\section{ACKNOWLEDGMENTS}

We would like to thank Georges Dôme for carefully reading the manuscript. One of us (G.R.) wishes to thank the SL-AP group at CERN for making this collaboration possible by inviting him to join the group for three months. We would also like to thank Francesco Ruggiero for constant encouragement and constructive criticism.

\section{APPENDIX A: CALCULATION OF THE COUPLING ANGLE IN THE $X$ - $Y$ PLANE}

The aim of this Appendix is to determine the angle $\theta$ by which the principal axes of the beam ellipse are tilted in the physical $(X-Y)$ plane.

Linear particle motion can be written as a superposition of the three eigenvectors $\vec{v}_{k}(k=I, I I, I I I)$

$$
\vec{X}(s)=\sum_{k=I, I I, I I I} \sqrt{J_{k}}\left[\overrightarrow{\boldsymbol{v}}_{k}(s) e^{-i \phi_{k}}+\overrightarrow{\boldsymbol{v}}_{k}^{*}(s) e^{i \phi_{k}}\right],
$$

with $\vec{X} \equiv\left(X, P_{X}, Y, P_{Y}, Z, P_{Z}\right)^{T}$. For the eigenvectors,

$$
\vec{v}_{k}(s)=M\left(s, s_{0}\right) \vec{v}_{k}\left(s_{0}\right)
$$

holds, with

$$
M\left(s_{0}+L, s_{0}\right) \overrightarrow{\boldsymbol{v}}_{k}\left(s_{0}\right)=e^{-i 2 \pi Q_{k}} \overrightarrow{\boldsymbol{v}}_{k}\left(s_{0}\right),
$$

where $L$ is the circumference of the accelerator and $Q_{k}$ is the tune for the $k$ th mode. They obey the orthogonality relations [5] $\left(\vec{v}_{k}^{+} \equiv\left(\vec{v}_{k}^{T}\right)^{*}\right.$

$$
\begin{aligned}
& \overrightarrow{\boldsymbol{v}}_{k}^{+}\left(s_{0}\right) \underline{\boldsymbol{J}} \overrightarrow{\boldsymbol{v}}_{k}\left(s_{0}\right)=-\overrightarrow{\boldsymbol{v}}_{-k}^{+}\left(s_{0}\right) \underline{\boldsymbol{J}} \overrightarrow{\boldsymbol{v}}_{-k}\left(s_{0}\right)=i, \\
& \overrightarrow{\boldsymbol{v}}_{\mu}^{+}\left(s_{0}\right) \underline{\boldsymbol{J}} \overrightarrow{\boldsymbol{v}}_{\nu}\left(s_{0}\right)=0, \quad \text { otherwise }
\end{aligned}
$$

with

$$
\vec{v}_{-k} \equiv \vec{v}_{k}^{*}
$$

and

$$
\underline{J}=\left(\begin{array}{cccccc}
0 & -1 & 0 & 0 & 0 & 0 \\
1 & 0 & 0 & 0 & 0 & 0 \\
0 & 0 & 0 & -1 & 0 & 0 \\
0 & 0 & 1 & 0 & 0 & 0 \\
0 & 0 & 0 & 0 & 0 & -1 \\
0 & 0 & 0 & 0 & 1 & 0
\end{array}\right) .
$$

In particular, one has

$$
\begin{gathered}
X=\sum_{k=I, I I, I I I} \sqrt{J_{k}}\left[v_{k 1} e^{-i \phi_{k}}+v_{k 1}^{*} e^{i \phi_{k}}\right], \\
Y=\sum_{k=I, I I, I I I} \sqrt{J_{k}}\left[v_{k 3} e^{-i \phi_{k}}+v_{k 3}^{*} e^{i \phi_{k}}\right] .
\end{gathered}
$$

The rotation of the coordinates in the physical plane are given by

$$
\tilde{X}=X \cos \theta+Y \sin \theta, \quad \tilde{Y}=-X \sin \theta+Y \cos \theta .
$$

The rotated horizontal coordinate $\tilde{X}$ reads

$$
\begin{aligned}
& \tilde{X}(\theta)=\sum_{k=I, I I, I I I} \sqrt{J_{k}}[ {\left[\left(v_{k 1} \cos \theta+v_{k 3} \sin \theta\right) e^{-i \phi_{k}}\right.} \\
&\left.+\left(v_{k 1}^{*} \cos \theta+v_{k 3}^{*} \sin \theta\right) e^{i \phi_{k}}\right] .
\end{aligned}
$$

Averaging over the phase angles $\phi_{k}$ we arrive at

$$
\begin{aligned}
& \frac{1}{2}\left\langle\tilde{X}^{2}(\theta)\right\rangle=\sum_{k=I, I I, I I I} J_{k}\{[ {\left[v_{k 1} \cos \theta+v_{k 3} \sin \theta\right] } \\
&\left.\times\left[v_{k 1}^{*} \cos \theta+v_{k 3}^{*} \sin \theta\right]\right\}
\end{aligned}
$$

leading to

$$
E_{h}^{2}(\theta)=E_{x}^{2} \cos ^{2} \theta+E_{y}^{2} \sin ^{2} \theta+E_{x} G_{x} \sin 2 \theta,
$$

and using Eq. (2.9) we obtain

$$
\begin{aligned}
E_{x}^{2} & =2 \sum_{k=I, I I, I I I} J_{k} v_{k 1} v_{k 1}^{*} \equiv \Sigma_{11}, \\
E_{y}^{2} & =2 \sum_{k=I, I I, I I I} J_{k} v_{k 3} v_{k 3}^{*} \equiv \Sigma_{33}, \\
E_{x} G_{x} & =E_{y} G_{y}=\sum_{k=I, I I, I I I} J_{k}\left[v_{k 1} v_{k 3}^{*}+v_{k 1}^{*} v_{k 3}\right] \equiv \Sigma_{13}, \\
E_{h}^{2}(\theta) & \equiv\left\langle\tilde{X}^{2}(\theta)\right\rangle .
\end{aligned}
$$

For the vertical plane a similar solution can be obtained,

$$
\begin{aligned}
E_{v}^{2}(\theta) & =E_{x}^{2} \sin ^{2} \theta+E_{y}^{2} \cos ^{2} \theta-E_{y} G_{y} \sin 2 \theta \\
& =E_{h}^{2}(\theta+\pi / 2) .
\end{aligned}
$$

$E_{h}(\theta), E_{v}(\theta)$ are the maxima of the least squared amplitudes of the particle motion in the $\theta$ direction.

The principal axes $E_{1}, E_{2}$ can be found by maximizing $E_{h}^{2}(\theta)-E_{v}^{2}(\theta)$. This yields $\theta_{p}$

$$
\tan 2 \theta_{p}=\frac{2 E_{x} G_{x}}{E_{x}^{2}-E_{y}^{2}} .
$$

Choosing

$$
\sin 2 \theta_{p}=\frac{2 E_{x} G_{x}}{\mathcal{N}}, \quad \cos 2 \theta_{p}=\frac{E_{x}^{2}-E_{y}^{2}}{\mathcal{N}},
$$

we obtain

$$
\begin{aligned}
& E_{h}^{2}\left(\theta_{p}\right)=E_{1}^{2}=\frac{1}{2}\left\{\left[E_{x}^{2}+E_{y}^{2}\right]+\mathcal{N}\right\} \equiv\left\langle\hat{X}^{2}\right\rangle, \\
& E_{v}^{2}\left(\theta_{p}\right)=E_{2}^{2}=\frac{1}{2}\left\{\left[E_{x}^{2}+E_{y}^{2}\right]-\mathcal{N}\right\} \equiv\left\langle\hat{Y}^{2}\right\rangle,
\end{aligned}
$$

where $\mathcal{N}= \pm \sqrt{\left[E_{x}^{2}-E_{y}^{2}\right]^{2}+4\left(E_{x} G_{x}\right)^{2}}$. The sign of $\mathcal{N}$ may be chosen in such a way that $\cos 2 \theta_{p}$ becomes positive,

$$
\mathcal{N}=\operatorname{sgn}\left(E_{x}^{2}-E_{y}^{2}\right) \sqrt{\left[E_{x}^{2}-E_{y}^{2}\right]^{2}+4\left(E_{x} G_{x}\right)^{2}},
$$




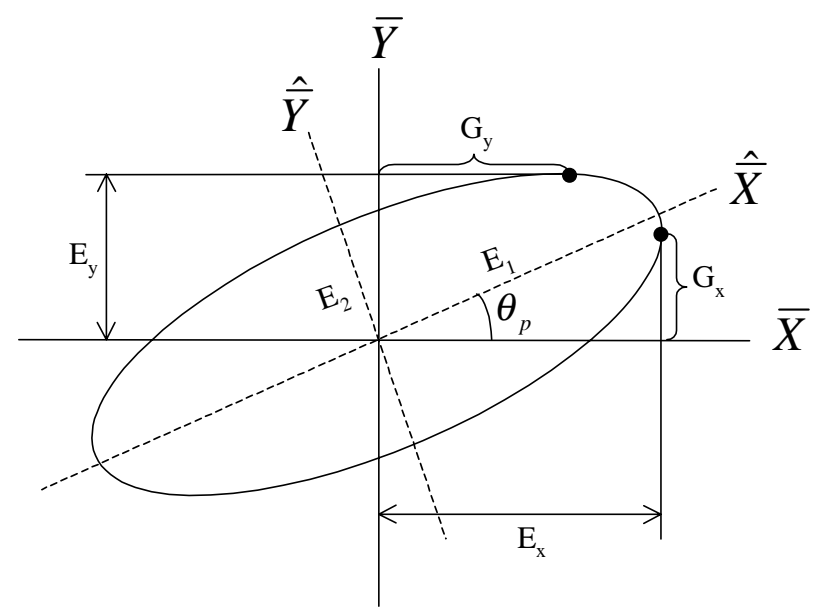

FIG. 2. Cross section of the averaged plane $(\bar{X}-\bar{Y})$.

i.e., $-\pi / 4 \leq \theta_{p} \leq \pi / 4$. The beam ellipse with respect to the principal axes $(\hat{\bar{X}}, \hat{\bar{Y}})$ can be written as

$$
\frac{\hat{\bar{X}}^{2}}{E_{1}^{2}}+\frac{\hat{\bar{Y}}^{2}}{E_{2}^{2}}=1 .
$$

Figure 2 illustrates the relation between $E_{x}, E_{y}, G_{x}, G_{y}$, $E_{1}, E_{2}$, and $\theta_{p}$.

Equation (18) can be rewritten for the averaged coordinates $\bar{X}$ and $\bar{Y}$,

$$
E_{y}^{2} \bar{X}^{2}-2 E_{x} G_{x} \bar{X} \bar{Y}+E_{x}^{2} \bar{Y}^{2}=E_{1}^{2} E_{2}^{2}=\epsilon^{2},
$$

with

$$
\epsilon^{2}=E_{x}^{2}\left(E_{y}^{2}-G_{x}^{2}\right)=E_{y}^{2}\left(E_{x}^{2}-G_{y}^{2}\right),
$$

which has the following solution using an arbitrary angle $\psi$ :

$$
\left(\begin{array}{c}
\bar{X}(\psi) \\
\bar{Y}(\psi)
\end{array}\right)=\left(\begin{array}{c}
E_{x} \\
G_{x}
\end{array}\right) \cos \psi+\left(\frac{0}{\sqrt{E_{y}^{2}-G_{x}^{2}}}\right) \sin \psi .
$$

Note that Eqs. (A19) and (A20), which define the beam cross section, are the result of a many particle treatment due to the averaging process described in Eq. (A10) (see Refs. [5-7]).

Last, we can rewrite $\tan 2 \theta_{p}$ as

$$
\begin{aligned}
& \sin \theta_{p}=\operatorname{sgn}\left\{\left(E_{x}^{2}-E_{y}^{2}\right) E_{x} G_{x}\right\} \sqrt{\frac{1}{2}\left(1-\cos 2 \theta_{p}\right)}, \\
& \cos \theta_{p}=\sqrt{\frac{1}{2}\left(1+\cos 2 \theta_{p}\right)} .
\end{aligned}
$$

\section{Coupling angle in the physical $X-Y$ plane}

Although the coupling angle is defined for a multiparticle system it is instructive to relate it to the physical plane $(X-Y)$ of single particle motion. In this plane, motion is restricted to a parallelogram which is tilted due to the linear coupling.

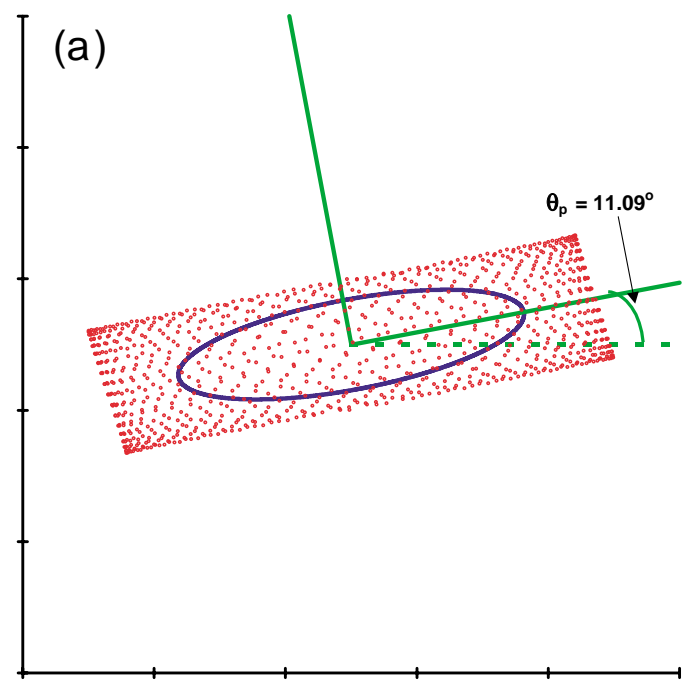

(b)
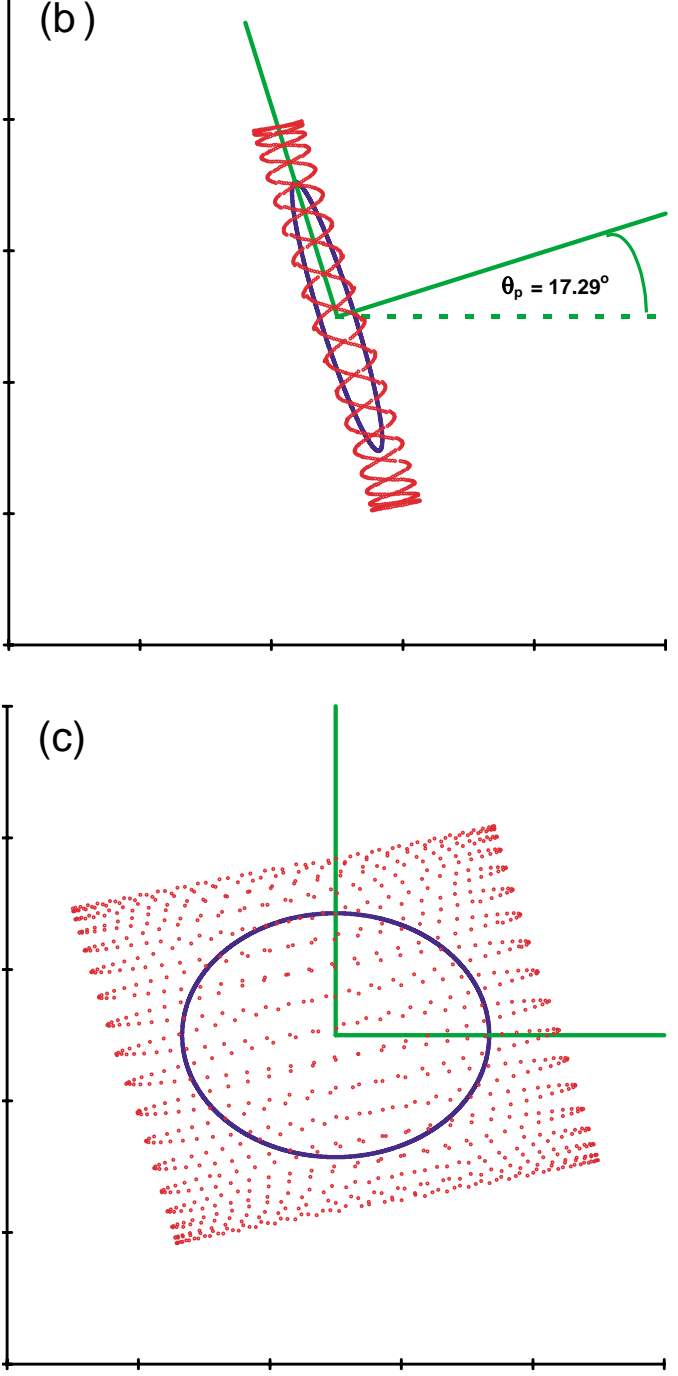

FIG. 3. (Color) Numerical calculation of the single particle motion in the $(X-Y)$ plane The action ratios between horizontal and vertical motion planes are $1: 10,10: 1$, and 1:1 in parts (a), (b), and (c), respectively. The angle $\theta_{p}$ is given in degrees. The ellipse represents the cross section of the averaged $(\bar{X}-\bar{Y})$ plane. 
Numerical simulations were carried out for a FODO structure including considerable skew quadrupole components. Three particles have been tracked over 1000 turns for mainly horizontal motion, mainly vertical motion, and for a $J_{I}=J_{I I}$ beam in Figs. 3(a), 3(b), and 3(c), respectively. In the limit of one dominant plane of motion [see Fig. 3(a) or 3(b)] the calculated coupling angle is the angle by which the longer side of the parallelogram is tilted. For the intermediate case the coupling angle goes to zero when the ellipse approaches the circle; see Fig. 3(c).

Note that a zero coupling angle does not imply that the motion is decoupled, but, instead, it means that the eigenplanes are not tilted with respect to the uncoupled case.

\section{APPENDIX B: SYNCHROBEAM MAPPING WITHIN SOLENOID FIELDS}

The synchrobeam map is described by Eq. (2.51). It consists of a transformation from the IP to the CP, followed by the beam-beam kick and the backwards transformation to the IP. In this Appendix the transformation $D(S)$ from the IP to the CP is generalized to include solenoid fields. For a particle within a solenoid field one obtains in linear approximation in $S$

$$
\begin{array}{ll}
\bar{x}^{*}=x^{*}+S \frac{\partial \mathcal{H}}{\partial p_{x}^{*}}, & \bar{p}_{x}^{*}=p_{x}^{*}-S \frac{\partial \mathcal{H}}{\partial x^{*}}, \\
\bar{y}^{*}=y^{*}+S \frac{\partial \mathcal{H}}{\partial p_{y}^{*}}, & \bar{p}_{y}^{*}=p_{y}^{*}-S \frac{\partial \mathcal{H}}{\partial y^{*}}, \\
\bar{z}^{*}=z^{*}+S \frac{\partial \mathcal{H}}{\partial p_{z}^{*}}, & \bar{p}_{z}^{*}=p_{z}^{*}-S \frac{\partial \mathcal{H}}{\partial z^{*}},
\end{array}
$$

with $S$ given by Eq. (2.44)

$$
S=S\left(z^{*}, Z^{\dagger}\right)=\frac{z^{*}-Z^{\dagger}}{2} \text {. }
$$

The normal coordinates are taken at the interaction point while the barred coordinates are taken at the collision point. In the following we skip the symbol $*$, which indicates the Lorentz transformation of the coordinates of the test particle. Note that we temporarily ignore the contribution of the strong beam [see Eq. (2.49)].

It is most convenient to symplectify Eq. (B1) such that the total SBM transformation is symplectic by definition. In the following, three methods of symplectification shall be described and applied by using the Hamiltonian of a solenoid field. The last method is evaluated for arbitrary energies.

\section{A. Generating function}

We introduce the generating function

$$
\begin{aligned}
F_{2}\left(x, \bar{p}_{x} ; y, \bar{p}_{y} ; z, \bar{p}_{z}\right)= & x \bar{p}_{x}+y \bar{p}_{y}+z \bar{p}_{z} \\
& +S \mathcal{H}\left(x, \bar{p}_{x} ; y, \bar{p}_{y} ; z, \bar{p}_{z}\right)
\end{aligned}
$$

in analogy to the method applied by Forest and Ohmi for the symplectic integration of complex wigglers $[8,9]$. The transformation equations due to $F_{2}$ take the form

$$
\begin{aligned}
& \bar{x}=\frac{\partial F_{2}}{\partial \bar{p}_{x}}=x+S \frac{\partial \mathcal{H}}{\partial \bar{p}_{x}}, \\
& p_{x}=\frac{\partial F_{2}}{\partial x}=\bar{p}_{x}+S \frac{\partial \mathcal{H}}{\partial x}, \\
& \bar{y}=\frac{\partial F_{2}}{\partial \bar{p}_{y}}=y+S \frac{\partial \mathcal{H}}{\partial \bar{p}_{y}}, \\
& p_{y}=\frac{\partial F_{2}}{\partial y}=\bar{p}_{y}+S \frac{\partial \mathcal{H}}{\partial y}, \\
& \bar{z}=\frac{\partial F_{2}}{\partial \bar{p}_{z}}=z+S \frac{\partial \mathcal{H}}{\partial \bar{p}_{z}}, \\
& p_{z}=\frac{\partial F_{2}}{\partial z}=\bar{p}_{z}+\frac{1}{2} \mathcal{H}+S \frac{\partial \mathcal{H}}{\partial z} .
\end{aligned}
$$

For a solenoid field (with strength $H$ ) the Hamiltonian reads

$$
\begin{aligned}
\mathcal{H}\left(x, p_{x} ; y, p_{y} ; z, p_{z}\right)=\frac{1}{2}\{ & {\left[p_{x}+y H\right]^{2} } \\
& \left.+\left[p_{y}-x H\right]^{2}\right\} .
\end{aligned}
$$

The corresponding generating function is

$$
\begin{aligned}
& F_{2}\left(x, \bar{p}_{x} ; y, \bar{p}_{y} ; z, \bar{p}_{z}\right)= x \bar{p}_{x}+y \bar{p}_{y}+z \bar{p}_{z} \\
&+\frac{S}{2}\left\{\left[\bar{p}_{x}+y H\right]^{2}\right. \\
&\left.+\left[\bar{p}_{y}-x H\right]^{2}\right\} .
\end{aligned}
$$

Thus,

$$
\begin{aligned}
\bar{x} & =\frac{\partial F_{2}}{\partial \bar{p}_{x}}=x+S\left[\bar{p}_{x}+y H\right] \\
p_{x} & =\frac{\partial F_{2}}{\partial x}=\bar{p}_{x}-S\left[\bar{p}_{y}-x H\right] H \\
\bar{y} & =\frac{\partial F_{2}}{\partial \bar{p}_{y}}=y+S\left[\bar{p}_{y}-x H\right] \\
p_{y}= & \frac{\partial F_{2}}{\partial y}=\bar{p}_{y}+S\left[\bar{p}_{x}+y H\right] H \\
\bar{z} & =\frac{\partial F_{2}}{\partial \bar{p}_{z}}=z \\
p_{z} & =\frac{\partial F_{2}}{\partial z}=\bar{p}_{z}+\frac{1}{4}\left\{\left[\bar{p}_{x}+y H\right]^{2}+\left[\bar{p}_{y}-x H\right]^{2}\right\}
\end{aligned}
$$

From Eqs. (B7) we get 


$$
\begin{array}{ll}
\bar{x}-S \bar{p}_{x}=x+S y H, & \bar{p}_{x}-S \bar{p}_{y} H=p_{x}-S x H^{2}, \\
\bar{y}-S \bar{p}_{y}=y-S x H, & \bar{p}_{y}+S \bar{p}_{x} H=p_{y}-S y H^{2},
\end{array}
$$

or

$$
\left(\begin{array}{cccc}
1 & -S & 0 & 0 \\
0 & 1 & 0 & -S H \\
0 & 0 & 1 & -S \\
0 & S H & 0 & 1
\end{array}\right)\left(\begin{array}{c}
\bar{x} \\
\bar{p}_{x} \\
\bar{y} \\
\bar{p}_{y}
\end{array}\right)=\left(\begin{array}{cccc}
1 & 0 & S H & 0 \\
-S H^{2} & 1 & 0 & 0 \\
-S H & 0 & 1 & 0 \\
0 & 0 & -S H^{2} & 1
\end{array}\right)\left(\begin{array}{c}
x \\
p_{x} \\
y \\
p_{y}
\end{array}\right)
$$

Using the relation

$$
\left(\begin{array}{cccc}
1 & -S & 0 & 0 \\
0 & 1 & 0 & -S H \\
0 & 0 & 1 & -S \\
0 & S H & 0 & 1
\end{array}\right)^{-1}=\frac{1}{1+(S H)^{2}}\left(\begin{array}{cccc}
1+S^{2} & S & 0 & S^{2} H \\
0 & 1 & 0 & S H \\
0 & -S^{2} H & 1+(S H)^{2} & S \\
0 & -S H & 0 & 1
\end{array}\right),
$$

we obtain from (B9)

$$
\left(\begin{array}{c}
\bar{x} \\
\bar{p}_{x} \\
\bar{y} \\
\bar{p}_{y}
\end{array}\right)=\frac{1}{1+(S H)^{2}}\left(\begin{array}{cccc}
1 & S & S H & S^{2} H \\
-S H^{2} & 1 & -S^{2} H^{3} & S H \\
-S H & -S^{2} H & 1 & S \\
S^{2} H^{3} & -S H & -S H^{2} & 1
\end{array}\right)\left(\begin{array}{c}
x \\
p_{x} \\
y \\
p_{y}
\end{array}\right) .
$$

In particular, we have

$$
\bar{p}_{x}+y H=\frac{S H\left[p_{y}-x H\right]+\left[p_{x}+y H\right]}{1+(S H)^{2}}, \quad \bar{p}_{y}-x H=\frac{-S H\left[p_{x}+y H\right]+\left[p_{y}-x H\right]}{1+(S H)^{2}} .
$$

Inserting Eqs. (B12) into (B7), we finally get

$$
\bar{p}_{z}=p_{z}-\frac{1}{4} \frac{\left[p_{x}+y H\right]^{2}+\left[p_{y}-x H\right]^{2}}{1+(S H)^{2}} .
$$

Note that $z$ remains unchanged [see Eq. (B7)].

Note that, for $H=0$ (drift space), we obtain from Eqs. (B7), (B11), and (B13) the transformation equations

$$
\begin{gathered}
\bar{x}=x+S p_{x}, \quad \bar{p}_{x}=p_{x}, \\
\bar{y}=y+S p_{y}, \quad \bar{p}_{y}=p_{y}, \\
\bar{z}=z, \quad \bar{p}_{z}=p_{z}-\frac{1}{4}\left(p_{x}^{2}+p_{y}^{2}\right),
\end{gathered}
$$

which are the same as Eqs. (2.49) and (2.50) without the extra terms due to the strong beam.

\section{B. Lie series}

In the following we again skip the symbol $*$ which indicates the Lorentz transformation of the coordinates of the test particle. The canonical equations of motion

$$
\begin{aligned}
\frac{d x}{d s}=\frac{\partial \mathcal{H}}{\partial p_{x}}, & \frac{d p_{x}}{d s}=-\frac{\partial \mathcal{H}}{\partial x}, \\
\frac{d y}{d s}=\frac{\partial \mathcal{H}}{\partial p_{y}}, & \frac{d p_{y}}{d s}=-\frac{\partial \mathcal{H}}{\partial y}, \\
\frac{d z}{d s}=\frac{\partial \mathcal{H}}{\partial p_{z}}, & \frac{d p_{z}}{d s}=-\frac{\partial \mathcal{H}}{\partial z},
\end{aligned}
$$

due to a Hamiltonian

$$
\mathcal{H}(\vec{x})=\mathcal{H}\left(x, p_{x}, y, p_{y}, z, p_{z}\right)
$$

can be integrated by Lie series $[10,11]$

$$
\overrightarrow{\bar{x}}(s)=\exp \left[\left(s-s_{0}\right) D\right] \vec{x}
$$

with

$$
\begin{aligned}
\vec{x} & =\left(x, p_{x}, y, p_{y}, z, p_{z}\right)^{T}, \\
\overrightarrow{\bar{x}} & =\left(\bar{x}, \bar{p}_{x}, \bar{y}, \bar{p}_{y}, \bar{z}, \bar{p}_{z}\right)^{T}, \\
\vec{x} & \equiv \vec{x}\left(s_{0}\right),
\end{aligned}
$$

and

$$
\begin{aligned}
D= & {\left[\frac{\partial}{\partial p_{x}} \mathcal{H}(\vec{x})\right] \frac{\partial}{\partial x}-\left[\frac{\partial}{\partial x} \mathcal{H}(\vec{x})\right] \frac{\partial}{\partial p_{x}} } \\
& +\left[\frac{\partial}{\partial p_{y}} \mathcal{H}(\vec{x})\right] \frac{\partial}{\partial y}-\left[\frac{\partial}{\partial y} \mathcal{H}(\vec{x})\right] \frac{\partial}{\partial p_{y}} \\
& +\left[\frac{\partial}{\partial p_{z}} \mathcal{H}(\vec{x})\right] \frac{\partial}{\partial z}-\left[\frac{\partial}{\partial z} \mathcal{H}(\vec{x})\right] \frac{\partial}{\partial p_{z}} .
\end{aligned}
$$

In particular, we get for the map from the IP to the CP $s_{\mathrm{CP}}=s_{\mathrm{IP}}+S$

$$
\overrightarrow{\bar{x}}\left(s_{\mathrm{CP}}\right)=\exp (S D) \vec{x}, \quad \vec{x} \equiv \vec{x}\left(s_{\mathrm{IP}}\right),
$$

which is not symplectic in general due to the factor $S$.

In order to symplectify Eq. (B20), we introduce a new Hamiltonian

$$
\mathcal{H}_{S B M}(\vec{x})=S \mathcal{H}(\vec{x})
$$


by modifying the longitudinal coordinates of motion, leading to the canonical map

$$
\overrightarrow{\bar{x}}\left(s_{\mathrm{CP}}\right)=\exp \left(D_{\mathrm{SBM}}\right) \vec{x}
$$

with

$$
\begin{aligned}
D_{\mathrm{SBM}}= & {\left[\frac{\partial}{\partial p_{x}} \mathcal{H}_{\mathrm{SBM}}(\vec{x})\right] \frac{\partial}{\partial x}-\left[\frac{\partial}{\partial x} \mathcal{H}_{\mathrm{SBM}}(\vec{x})\right] \frac{\partial}{\partial p_{x}}+\left[\frac{\partial}{\partial p_{y}} \mathcal{H}_{\mathrm{SBM}}(\vec{x})\right] \frac{\partial}{\partial y}-\left[\frac{\partial}{\partial y} \mathcal{H}_{\mathrm{SBM}}(\vec{x})\right] \frac{\partial}{\partial p_{y}} } \\
& +\left[\frac{\partial}{\partial p_{z}} \mathcal{H}_{\mathrm{SBM}}(\vec{x})\right] \frac{\partial}{\partial z}-\left[\frac{\partial}{\partial z} \mathcal{H}_{\mathrm{SBM}}(\vec{x})\right] \frac{\partial}{\partial p_{z}} \\
= & S D-\frac{1}{2} \mathcal{H}(\vec{x}) \frac{\partial}{\partial p_{z}}
\end{aligned}
$$

and $D$ given by Eq. (B19).

Using the Hamiltonian

$$
\mathcal{H}(\vec{x})=\frac{1}{2}\left\{\left[p_{x}+y H\right]^{2}+\left[p_{y}-x H\right]^{2}\right\}
$$

of a solenoid, we obtain

$$
\begin{aligned}
& D=\left[p_{x}+y H\right] \frac{\partial}{\partial x}+\left[p_{y}-x H\right] H \frac{\partial}{\partial p_{x}} \\
& +\left[p_{y}-x H\right] \frac{\partial}{\partial y}-\left[p_{x}+y H\right] H \frac{\partial}{\partial p_{y}}, \\
& D_{\mathrm{SBM}}=S D-\frac{1}{4}\left\{\left[p_{x}+y H\right]^{2}+\left[p_{y}-x H\right]^{2}\right\} \frac{\partial}{\partial p_{z}} \text {. } \\
& D_{\mathrm{SBM}} p_{z}=-\frac{1}{2} \mathcal{H}(\vec{x}) \\
& =-\frac{1}{4}\left\{\left[p_{x}+y H\right]^{2}+\left[p_{y}-x H\right]^{2}\right\},
\end{aligned}
$$

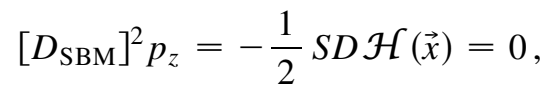

$$
\begin{aligned}
& {\left[D_{\mathrm{SBM}}\right]^{\nu} p_{z}=0 \text { for } \nu>1 \text {, }}
\end{aligned}
$$

resulting in

$$
\left\{\exp \left[D_{S B M}\right]\right\} z=z
$$

and

For the longitudinal coordinates we then get

$$
\begin{aligned}
& D_{\mathrm{SBM}} z=S D z=0, \\
& {\left[D_{\mathrm{SBM}}\right]^{\nu} z=0 \text { for } \nu \in \mathbb{N} \text {, }} \\
& \begin{array}{l}
\Longrightarrow\left\{\exp \left[D_{\mathrm{SBM}}\right]\right\} p_{z} \\
=p_{z}-\frac{1}{4}\left\{\left[p_{x}+y H\right]^{2}+\left[p_{y}-x H\right]^{2}\right\}
\end{array}
\end{aligned}
$$

For the transverse coordinates we have

$$
\begin{aligned}
& D_{\mathrm{SBM}}\left(\begin{array}{c}
x \\
p_{x} \\
y \\
p_{y}
\end{array}\right)=S\left(\begin{array}{c}
p_{x}+y H \\
p_{y} H-x H^{2} \\
p_{y}-x H \\
-p_{x} H-y H^{2}
\end{array}\right)=S \underbrace{\left(\begin{array}{cccc}
0 & 1 & H & 0 \\
-H^{2} & 0 & 0 & H \\
-H & 0 & 0 & 1 \\
0 & -H & -H^{2} & 0
\end{array}\right)}_{\underline{\mathrm{C}}}\left(\begin{array}{c}
x \\
p_{x} \\
y \\
p_{y}
\end{array}\right) \\
& {\left[D_{\mathrm{SBM}}\right]^{\nu}\left(\begin{array}{c}
x \\
p_{x} \\
y \\
p_{y}
\end{array}\right)=S^{\nu} D^{\nu}\left(\begin{array}{c}
x \\
p_{x} \\
y \\
p_{y}
\end{array}\right)=S^{\nu} \underline{C}^{\nu}\left(\begin{array}{c}
x \\
p_{x} \\
y \\
p_{y}
\end{array}\right) \Longrightarrow\left\{\exp \left[D_{\mathrm{SBM}}\right]\right\}\left(\begin{array}{c}
x \\
p_{x} \\
y \\
p_{y}
\end{array}\right)=\{\exp [S \underline{C}]\}\left(\begin{array}{c}
x \\
p_{x} \\
y \\
p_{y}
\end{array}\right) .}
\end{aligned}
$$

In order to calculate $\exp [S \underline{C}]$, we write

$$
\underline{C}=\underline{C}_{1}+\underline{C}_{2}
$$

with

$$
\underline{C}_{1}=\left(\begin{array}{cccc}
0 & 1 & 0 & 0 \\
-H^{2} & 0 & 0 & 0 \\
0 & 0 & 0 & 1 \\
0 & 0 & -H^{2} & 0
\end{array}\right), \quad \underline{C}_{2}=H\left(\begin{array}{cccc}
0 & 0 & 1 & 0 \\
0 & 0 & 0 & 1 \\
-1 & 0 & 0 & 0 \\
0 & -1 & 0 & 0
\end{array}\right)
$$


and

$$
\underline{C}_{1} \cdot \underline{C}_{2}=\underline{C}_{2} \cdot \underline{C}_{1} \Longrightarrow \exp [S \underline{C}]=\exp \left[S \underline{C}_{1}\right] \exp \left[S \underline{C}_{2}\right]
$$

Furthermore, we obtain

$$
\left[\underline{C}_{1}\right]^{2 n}=(-1)^{n} H^{2 n} \underline{I}, \quad\left[\underline{C}_{1}\right]^{2 n+1}=(-1)^{n} H^{2 n} \underline{C}_{1}=(-1)^{n} H^{2 n+1}\left(\begin{array}{cccc}
0 & 1 / H & 0 & 0 \\
-H & 0 & 0 & 0 \\
0 & 0 & 0 & 1 / H \\
0 & 0 & -H & 0
\end{array}\right)
$$

(with $\underline{I}$ a $4 \times 4$ unity matrix), and thus

$$
\begin{aligned}
\exp \left[S \underline{C}_{1}\right] & =\sum_{n=0}^{\infty} \frac{1}{(2 n) !}(-1)^{n}(\Delta \theta)^{2 n} \underline{I}+\sum_{n=0}^{\infty} \frac{1}{(2 n+1) !}(-1)^{n}(\Delta \theta)^{2 n+1}\left(\begin{array}{cccc}
0 & 1 / H & 0 & 0 \\
-H & 0 & 0 & 0 \\
0 & 0 & 0 & 1 / H \\
0 & 0 & -H & 0
\end{array}\right) \\
& =\underline{I} \cos \Delta \theta+\left(\begin{array}{cccc}
0 & 1 / H & 0 & 0 \\
-H & 0 & 0 & 0 \\
0 & 0 & 0 & 1 / H \\
0 & 0 & -H & 0
\end{array}\right) \sin \Delta \theta
\end{aligned}
$$

resulting in

$$
\exp \left[S \underline{C}_{1}\right]=\left(\begin{array}{cccc}
\cos \Delta \theta & \frac{\sin \Delta \theta}{H} & 0 & 0 \\
-H \sin \Delta \theta & \cos \Delta \theta & 0 & 0 \\
0 & 0 & \cos \Delta \theta & \frac{\sin \Delta \theta}{H} \\
0 & 0 & -H \sin \Delta \theta & \cos \Delta \theta
\end{array}\right)
$$

with $\Delta \theta=S H$.

In a similar way one can derive

$$
\left[\underline{C}_{2}\right]^{2 n}=(-1)^{n} H^{2 n} \underline{I}, \quad\left[\underline{C}_{2}\right]^{2 n+1}=(-1)^{n} H^{2 n+1}\left[\frac{1}{H} \underline{C}_{2}\right],
$$

yielding

$$
\exp \left[S \underline{C}_{2}\right]=\underline{I} \cos \Delta \theta+\left[\frac{1}{H} \underline{C}_{2}\right] \sin \Delta \theta=\left(\begin{array}{cccc}
\cos \Delta \theta & 0 & \sin \Delta \theta & 0 \\
0 & \cos \Delta \theta & 0 & \sin \Delta \theta \\
-\sin \Delta \theta & 0 & \cos \Delta \theta & 0 \\
0 & -\sin \Delta \theta & 0 & \cos \Delta \theta
\end{array}\right) .
$$

Inserting Eqs. (B37) and (B39) into Eq. (B34), we finally obtain [see Eq. (B31)]

$$
\begin{aligned}
\left(\begin{array}{c}
\bar{x} \\
\overline{p_{x}} \\
\bar{y} \\
\overline{p_{y}}
\end{array}\right) & =\left\{\exp \left[D_{\mathrm{SBM}}\right]\right\}\left(\begin{array}{c}
x \\
p_{x} \\
y \\
p_{y}
\end{array}\right) \\
& =\left(\begin{array}{cccc}
\cos \Delta \theta & \frac{\sin \Delta \theta}{H} & 0 & 0 \\
-H \sin \Delta \theta & \cos \Delta \theta & 0 & 0 \\
0 & 0 & \cos \Delta \theta & \frac{\sin \Delta \theta}{H} \\
0 & 0 & -H \sin \Delta \theta & \cos \Delta \theta
\end{array}\right)\left(\begin{array}{cccc}
\cos \Delta \theta & 0 & \sin \Delta \theta & 0 \\
0 & \cos \Delta \theta & 0 & \sin \Delta \theta \\
-\sin \Delta \theta & 0 & \cos \Delta \theta & 0 \\
0 & -\sin \Delta \theta & 0 & \cos \Delta \theta
\end{array}\right)\left(\begin{array}{c}
x \\
p_{x} \\
y \\
p_{y}
\end{array}\right) .
\end{aligned}
$$

Equations (B11) and (B40) are both symplectic approximations of the solenoid kick. The approximations agree in first-order with respect to $\Delta \theta=S H$. In particular, in the limit of $H=0$, which represents a drift space, they give the same results [see Eq. (B14)].

\section{Integration method}

Moreover, we will generalize the function for arbitrary energy. The map $\vec{x}\left(s_{\mathrm{IP}}\right) \rightarrow \overrightarrow{\bar{x}}\left(s_{\mathrm{CP}}\right)$ can also be obtained by introducing an artificial parameter $\tau$ and solving the canonical equations of motion

$$
\vec{x}^{\prime} \equiv \frac{d}{d \tau} \vec{x}=-\underline{J} \frac{\partial}{\partial \vec{x}} \mathcal{H}_{\mathrm{SBM}}
$$

for $\Delta \tau=1$ with $\underline{J}$ given by (A6) [see Eqs. (B17) and (B22)].

We demonstrate this by using the new Hamiltonian (again denoted by $\mathcal{H}$ ) 


$$
\mathcal{H}=\frac{\mathcal{H}_{0}\left(x, p_{x}, y, p_{y}\right)}{\left[1+p_{z}\right]}
$$

with

$$
\mathcal{H}_{0}\left(x, p_{x} ; y, p_{y}\right)=\frac{1}{2}\left\{\left[p_{x}+y H\right]^{2}+\left[p_{y}-x H\right]^{2}\right\},
$$

thus generalizing the Hamiltonian $\mathcal{H}$ in Eqs. (B5) and (B24) by taking into account an arbitrary energy of the particles [due to the denominator $\left[1+p_{z}\right.$ ] in Eq. (B41)].

Then we have

$$
\mathcal{H}_{\mathrm{SBM}}=S \frac{\mathcal{H}_{0}\left(x, p_{x}, y, p_{y}\right)}{\left[1+p_{z}\right]}
$$

leading to the equations of motion

$$
\begin{aligned}
x^{\prime} & =\frac{\partial \mathcal{H}_{\mathrm{SBM}}}{\partial p_{x}}=S \frac{\left[p_{x}+y H\right]}{\left[1+p_{z}\right]}, \\
p_{x}^{\prime} & =-\frac{\partial \mathcal{H}_{\mathrm{SBM}}}{\partial x}=S \frac{\left[p_{y}-x H\right] H}{\left[1+p_{z}\right]}, \\
y^{\prime} & =\frac{\partial \mathcal{H}_{\mathrm{SBM}}}{\partial p_{y}}=S \frac{\left[p_{y}-x H\right]}{\left[1+p_{z}\right]}, \\
p_{y}^{\prime} & =-\frac{\partial \mathcal{H}_{\mathrm{SBM}}}{\partial y}=-S \frac{\left[p_{x}+y H\right] H}{\left[1+p_{z}\right]}, \\
z^{\prime} & =\frac{\partial \mathcal{H}_{\mathrm{SBM}}}{\partial p_{z}} \\
& =-\frac{S}{2} \frac{\left[p_{x}+y H\right]^{2}+\left[p_{y}-x H\right]^{2}}{\left[1+p_{z}\right]^{2}}, \\
p_{z}^{\prime} & =-\frac{\partial \mathcal{H}_{\mathrm{SBM}}}{\partial z} \\
& =-\frac{1}{4} \frac{\left[p_{x}+y H\right]^{2}+\left[p_{y}-x H\right]^{2}}{\left[1+p_{z}\right]} .
\end{aligned}
$$

From Eqs. (B45) we get

$$
\begin{aligned}
\frac{d}{d \tau} \mathcal{H}_{0}\left(x, p_{x}, y, p_{y}\right)= & \frac{\partial \mathcal{H}_{0}}{\partial x} x^{\prime}+\frac{\partial \mathcal{H}_{0}}{\partial p_{x}} p_{x}^{\prime} \\
& +\frac{\partial \mathcal{H}_{0}}{\partial y} y^{\prime}+\frac{\partial \mathcal{H}_{0}}{\partial p_{y}} p_{y}^{\prime} \\
= & 0 \\
& \Longrightarrow \mathcal{H}_{0}\left(x, p_{x}, y, p_{y}\right) \\
& =\text { const. }
\end{aligned}
$$

Thus Eq. (B45) takes the initial value

$$
p_{z}^{\prime}=-\frac{1}{2} \frac{\mathcal{H}_{0}\left(x, p_{x}, y, p_{y}\right)}{\left[1+p_{z}\right]}
$$

with the solution

$$
\begin{aligned}
\bar{p}_{z}(\tau) & =\left[1+p_{z}\right] \sqrt{1-\frac{1}{2} \frac{\mathcal{H}_{0}\left(x, p_{x}, y, p_{y}\right)}{\left[1+p_{z}\right]^{2}}} \tau-1 \\
\Longrightarrow & \bar{p}_{z}=\left[1+p_{z}\right] \sqrt{1-\frac{1}{2} \frac{\mathcal{H}_{0}\left(x, p_{x}, y, p_{y}\right)}{\left[1+p_{z}\right]^{2}}}-1,
\end{aligned}
$$

where we have used

$$
p_{z} \equiv p_{z}(0), \quad \bar{p}_{z} \equiv p_{z}(1) .
$$

From Eqs. (B45) we obtain

$$
\begin{aligned}
\frac{d}{d \tau} \frac{z-Z^{\dagger}}{\left[1+p_{z}\right]} & =\frac{z^{\prime}\left(1+p_{z}\right)-\left(z-Z^{\dagger}\right) p_{z}^{\prime}}{\left[1+p_{z}\right]^{2}}=0 \\
& \Longrightarrow \frac{\bar{z}-Z^{\dagger}}{\left[1+p_{z}\right]}=\frac{z(0)-Z^{\dagger}}{\left[1+p_{z}(0)\right]} \\
& \equiv \frac{z-Z^{\dagger}}{\left[1+p_{z}\right]},
\end{aligned}
$$

and thus after the synchrobeam mapping we get

$$
\bar{z}-Z^{\dagger}=\left(z-Z^{\dagger}\right) \sqrt{1-\frac{1}{2} \frac{\mathcal{H}_{0}\left(x, p_{x}, y, p_{y}\right)}{\left[1+p_{z}\right]^{2}}} .
$$

Finally, Eqs. (B45) can be written as

$$
\begin{aligned}
& x^{\prime}=\frac{1}{2} \frac{z-Z^{\dagger}}{\left[1+p_{z}\right]}\left[p_{x}+y H\right], \\
& p_{x}^{\prime}=\frac{1}{2} \frac{z-Z^{\dagger}}{\left[1+p_{z}\right]}\left[p_{y}-x H\right] H, \\
& y^{\prime}=\frac{1}{2} \frac{z-Z^{\dagger}}{\left[1+p_{z}\right]}\left[p_{y}-x H\right], \\
& p_{y}^{\prime}=-\frac{1}{2} \frac{z-Z^{\dagger}}{\left[1+p_{z}\right]}\left[p_{x}+y H\right] H,
\end{aligned}
$$

leading again to Eq. (B40) for $\Delta \tau=1$ and $\Delta \theta=$ $S H /\left(1+p_{z}\right)$.

\section{APPENDIX C: LINEAR BEAM-BEAM MODEL}

In this appendix we derive the linear beam-beam model for a single slice. Using Eq. (1.1) for the whole bunch at the IP, we can write [using Eq. (2.2)] 


$$
\begin{aligned}
& p_{x}=-N^{*} \frac{\partial}{\partial x} U\left(\hat{x}, \hat{y} ; \hat{\Sigma}_{11}, \hat{\Sigma}_{33}\right)=-N^{*}\left\{\cos \theta \frac{\partial}{\partial \hat{x}} U\left(\hat{x}, \hat{y} ; \hat{\Sigma}_{11}, \hat{\Sigma}_{33}\right)-\sin \theta \frac{\partial}{\partial \hat{y}} U\left(\hat{x}, \hat{y} ; \hat{\Sigma}_{11}, \hat{\Sigma}_{33}\right)\right\}, \\
& p_{y}=-N^{*} \frac{\partial}{\partial y} U\left(\hat{x}, \hat{y} ; \hat{\Sigma}_{11}, \hat{\Sigma}_{33}\right)=-N^{*}\left\{\sin \theta \frac{\partial}{\partial \hat{x}} U\left(\hat{x}, \hat{y} ; \hat{\Sigma}_{11}, \hat{\Sigma}_{33}\right)+\cos \theta \frac{\partial}{\partial \hat{y}} U\left(\hat{x}, \hat{y} ; \hat{\Sigma}_{11}, \hat{\Sigma}_{33}\right)\right\} .
\end{aligned}
$$

For small values

$$
\hat{x}^{2} \ll \hat{\Sigma}_{11}, \quad \hat{y}^{2} \ll \hat{\Sigma}_{33}
$$

the behavior is linear [12],

$$
\begin{aligned}
& N^{*} \frac{\partial}{\partial \hat{x}} U\left(\hat{x}, \hat{y} ; \hat{\Sigma}_{11}, \hat{\Sigma}_{33}\right)=\frac{1}{f_{1}} \hat{x}, \\
& N^{*} \frac{\partial}{\partial \hat{y}} U\left(\hat{x}, \hat{y} ; \hat{\Sigma}_{11}, \hat{\Sigma}_{33}\right)=\frac{1}{f_{2}} \hat{y},
\end{aligned}
$$

with focal length $f_{1}$ and $f_{2}$ defined by

$$
\begin{aligned}
& \frac{1}{f_{1}}=\frac{2 N^{*} r_{p}}{\gamma_{0}\left(E_{1}+E_{2}\right) E_{1}}, \\
& \frac{1}{f_{2}}=\frac{2 N^{*} r_{p}}{\gamma_{0}\left(E_{1}+E_{2}\right) E_{2}},
\end{aligned}
$$

and with $E_{1}$ and $E_{2}$ taken from Appendix A [Eq. (A16)]. Thus,

$$
\begin{aligned}
p_{x} & =-\hat{x} \frac{1}{f_{1}} \cos \theta+\hat{y} \frac{1}{f_{2}} \sin \theta=-\frac{[x \cos \theta+y \sin \theta]}{f_{1}} \cos \theta+\frac{[-x \sin \theta+y \cos \theta]}{f_{2}} \sin \theta \\
& =-\left(\frac{\cos ^{2} \theta}{f_{1}}+\frac{\sin ^{2} \theta}{f_{2}}\right) x-\frac{1}{2}\left(\frac{1}{f_{1}}-\frac{1}{f_{2}}\right) y \sin 2 \theta \\
p_{y} & =-\hat{x} \frac{1}{f_{1}} \sin \theta-\hat{y} \frac{1}{f_{2}} \cos \theta=-\frac{[x \cos \theta+y \sin \theta]}{f_{1}} \sin \theta-\frac{[-x \sin \theta+y \cos \theta]}{f_{2}} \cos \theta \\
& =-\frac{1}{2}\left(\frac{1}{f_{1}}-\frac{1}{f_{2}}\right) x \sin 2 \theta-\left(\frac{\sin ^{2} \theta}{f_{1}}+\frac{\cos ^{2} \theta}{f_{2}}\right) y .
\end{aligned}
$$

In matrix form we may write

$$
\vec{x}\left(s_{\mathrm{IP}}+0\right)=\underline{T}_{b b} \vec{x}\left(s_{\mathrm{IP}}-0\right)
$$

with

$$
\underline{T}_{b b}=\left(\begin{array}{cccccc}
1 & 0 & 0 & 0 & 0 & 0 \\
-F_{1} & 1 & -F & 0 & 0 & 0 \\
0 & 0 & 1 & 0 & 0 & 0 \\
-F & 0 & -F_{2} & 1 & 0 & 0 \\
0 & 0 & 0 & 0 & 1 & 0 \\
0 & 0 & 0 & 0 & 0 & 1
\end{array}\right)
$$

and

$$
\begin{gathered}
F_{1}=\frac{1}{f_{1}} \cos ^{2} \theta+\frac{1}{f_{2}} \sin ^{2} \theta, \\
F_{2}=\frac{1}{f_{1}} \sin ^{2} \theta+\frac{1}{f_{2}} \cos ^{2} \theta, \\
F=\frac{1}{2}\left(\frac{1}{f_{1}}-\frac{1}{f_{2}}\right) \sin 2 \theta .
\end{gathered}
$$

The equations of motion (C6) can be obtained from the Hamiltonian

$\mathcal{H}_{b b}=\left\{\frac{1}{2} F_{1} x^{2}+\frac{1}{2} F_{2} y^{2}+F x y\right\} \delta\left(s-s_{\mathrm{IP}}\right)$.

Note that $\underline{T}_{b b}$ in (C7) contains quadrupole components $\left(F_{1}\right.$ and $\left.F_{2}\right)$, focusing in both transverse planes. In addition there appears a skew quadrupole component $(F)$ resulting from the rotation angle $\theta$ of the cross section due to the strong beam ( $F$ vanishes for $\theta=0)$.

\section{APPENDIX D: DISPERSION FORMALISM INCLUDING THE BEAM-BEAM KICK}

\section{A. Canonical transformation}

The Hamiltonian for the whole ring consisting of bending magnets, quadrupoles, skew quadrupoles, and solenoids, including the beam-beam kick in linear form, reads

$$
\mathcal{H}=\mathcal{H}_{0}+\mathcal{H}_{b b}
$$

with $[13,14]$

$$
\begin{aligned}
\mathcal{H}_{0}\left(x, y, z ; p_{x}, p_{y}, p_{z} ; s\right)= & \frac{1}{2} \frac{1}{\gamma_{0}^{2}} p_{z}^{2}-\left[x K_{x}+y K_{y}\right] p_{z}+\frac{1}{2}\left\{\left[p_{x}+y H\right]^{2}+\left[p_{y}-x H\right]^{2}\right\} \\
& +\frac{1}{2}\left\{\left[K_{x}^{2}+g\right] x^{2}+\left[K_{y}^{2}-g\right] y^{2}-2 N x y\right\}-\frac{1}{2} z^{2} \frac{1}{\beta_{0}^{2}} \frac{e V(s)}{E_{0}} h \frac{2 \pi}{L} \cos \varphi_{\mathrm{rf}}
\end{aligned}
$$


( $h$ is the harmonic number, $V(s)$ is the rf voltage of the cavity, and $\varphi_{\mathrm{rf}}$ is the rf phase), where the following abbreviations have been introduced:

$$
\begin{aligned}
g & =\frac{e}{p_{0} c}\left(\frac{\partial \mathcal{B}_{y}}{\partial x}\right)_{x=y=0}, \\
N & =\frac{1}{2} \frac{e}{p_{0} c}\left(\frac{\partial \mathcal{B}_{x}}{\partial x}-\frac{\partial \mathcal{B}_{y}}{\partial y}\right)_{x=y=0}, \\
H & =\frac{1}{2} \frac{e}{p_{0} c} \mathcal{B}_{s}(0,0, s) \\
K_{x} & =\frac{e}{p_{0} c} \mathcal{B}_{y}(0,0, s) \\
K_{y} & =-\frac{e}{p_{0} c} \mathcal{B}_{x}(0,0, s) .
\end{aligned}
$$

Note that the second term of the Hamiltonian is due to solenoid fields $(H)$ which have been treated in Appendix B. $\mathcal{H}_{b b}$ is given by Eq. (C9) in Appendix C. The Hamiltonian (D1) then leads to the canonical equations of motion

$$
\begin{aligned}
\frac{d}{d s} x= & p_{x}+y H \\
\frac{d}{d s} p_{x}= & p_{z} K_{x}-\left[K_{x}^{2}+g\right] x+N y \\
& +\left[p_{y}-x H\right] H-\left[x F_{1}+y F\right] \delta\left(s-s_{\mathrm{IP}}\right), \\
\frac{d}{d s} y= & p_{y}-x H, \\
\frac{d}{d s} p_{y}= & p_{z} K_{y}-\left[K_{y}^{2}-g\right] y+N x \\
& -\left[p_{x}+y H\right] H-\left[y F_{2}+x F\right] \delta\left(s-s_{\mathrm{IP}}\right), \\
\frac{d}{d s} z= & -\left[x K_{x}+y K_{y}\right]+\frac{1}{\gamma_{0}^{2}} p_{z}, \\
\frac{d}{d s} p_{z}= & \frac{1}{\beta_{0}^{2}} z \frac{e V(s)}{E_{0}} h \frac{2 \pi}{L} \cos \varphi_{\mathrm{rf}} .
\end{aligned}
$$

Note that the linear transverse betatron oscillations and the longitudinal motion [Eqs. (D4)] are coupled by the terms

$$
p_{z} K_{x}, \quad p_{z} K_{y}, \quad \text { and } \quad-\left[x K_{x}+y K_{y}\right]
$$

respectively, i.e., depending on the curvature of the orbit in the bending magnets.

In order to simplify these equations we introduce dispersion

$$
\vec{D}(s)=\left(\begin{array}{c}
D_{1}(s) \\
D_{2}(s) \\
D_{3}(s) \\
D_{4}(s)
\end{array}\right), \quad \vec{D}(s)=\vec{D}(s+L) .
$$

New variables $\tilde{x}, \tilde{p}_{x}, \tilde{y}, \tilde{p}_{y}, \tilde{z}, \tilde{p}_{z}$ can be introduced which satisfy the dispersion relation

$$
\begin{array}{ll}
\tilde{x}=x-p_{z} D_{1}, & \tilde{p}_{x}=p_{x}-p_{z} D_{2}, \\
\tilde{y}=y-p_{z} D_{3}, & \tilde{p}_{y}=p_{y}-p_{z} D_{4} .
\end{array}
$$

This replacement

$$
\left(x, p_{x}, y, p_{y}, z, p_{z}\right) \longrightarrow\left(\tilde{x}, \tilde{p}_{x}, \tilde{y}, \tilde{p}_{y}, \tilde{z}, \tilde{p}_{z}\right)
$$

can be achieved using the generating function [14-17]

$$
\begin{aligned}
F_{2}\left(x, y, z, \tilde{p}_{x}, \tilde{p}_{y}, \tilde{p}_{z}\right)= & \tilde{p}_{x}\left[x-\tilde{p}_{z} D_{1}\right]+\tilde{p}_{z} x D_{2} \\
& +\tilde{p}_{y}\left[y-\tilde{p}_{z} D_{3}\right]+\tilde{p}_{z} y D_{4} \\
& -\frac{1}{2}\left[D_{1} D_{2}+D_{3} D_{4}\right] \tilde{p}_{z}^{2} \\
& +\tilde{p}_{z} z,
\end{aligned}
$$

with the result that

$$
\begin{aligned}
\tilde{x} & =\frac{\partial F_{2}}{\partial \tilde{p}_{x}}=x-\tilde{p}_{z} D_{1}, \quad p_{x}=\frac{\partial F_{2}}{\partial x}=\tilde{p}_{x}+\tilde{p}_{z} D_{2}, \\
\tilde{y} & =\frac{\partial F_{2}}{\partial \tilde{p}_{y}}=y-\tilde{p}_{z} D_{3}, \quad p_{y}=\frac{\partial F_{2}}{\partial y}=\tilde{p}_{y}+\tilde{p}_{z} D_{4}, \\
\tilde{z} & =\frac{\partial F_{2}}{\partial \tilde{p}_{z}}=z+\left[-\tilde{p}_{x} D_{1}+x D_{2}-\tilde{p}_{y} D_{3}+y D_{4}\right]-\left[D_{1} D_{2}+D_{3} D_{4}\right] \tilde{p}_{z} \\
& =z+\left\{-\tilde{p}_{x} D_{1}+\left[x-D_{1} \tilde{p}_{z}\right] D_{2}-\tilde{p}_{y} D_{3}+\left[y-D_{3} \tilde{p}_{z}\right] D_{4}\right\} \\
& =z+\left[-\tilde{p}_{x} D_{1}+\tilde{x} D_{2}-\tilde{p}_{y} D_{3}+\tilde{y} D_{4}\right]=z+\left[-p_{x} D_{1}+x D_{2}-p_{y} D_{3}+y D_{4}\right], \\
p_{z} & =\frac{\partial F_{2}}{\partial z}=\tilde{p}_{z},
\end{aligned}
$$


and

with

$$
\tilde{\mathcal{H}}=\mathcal{H}+\frac{\partial F_{2}}{\partial s}
$$

In matrix form, Eqs. (D10) read

$$
\overrightarrow{\tilde{x}}=\underline{K} \vec{x}, \quad \vec{x}=\underline{K}^{-1} \overrightarrow{\tilde{x}},
$$

$$
\overrightarrow{\tilde{x}}=\left(\begin{array}{c}
\tilde{x} \\
\tilde{p}_{x} \\
\tilde{y} \\
\tilde{p}_{y} \\
\tilde{z} \\
\tilde{p}_{z}
\end{array}\right)
$$

and

$$
\underline{K}(s)=\left(\begin{array}{cccccc}
1 & 0 & 0 & 0 & 0 & -D_{1} \\
0 & 1 & 0 & 0 & 0 & -D_{2} \\
0 & 0 & 1 & 0 & 0 & -D_{3} \\
0 & 0 & 0 & 1 & 0 & -D_{4} \\
D_{2} & -D_{1} & D_{4} & -D_{3} & 1 & 0 \\
0 & 0 & 0 & 0 & 0 & 1
\end{array}\right), \quad \underline{K}^{-1}(s)=\left(\begin{array}{cccccc}
1 & 0 & 0 & 0 & 0 & D_{1} \\
0 & 1 & 0 & 0 & 0 & D_{2} \\
0 & 0 & 1 & 0 & 0 & D_{3} \\
0 & 0 & 0 & 1 & 0 & D_{4} \\
-D_{2} & D_{1} & -D_{4} & D_{3} & 1 & 0 \\
0 & 0 & 0 & 0 & 0 & 1
\end{array}\right) .
$$

Note that $\underline{K}(s)$ is symplectic,

$$
\underline{K}^{T}(s) \underline{J} \underline{K}(s)=\underline{J}
$$

[with $\underline{J}$ given by Eq. (A6)].

Taking into account the defining equations for the dispersion in the general case of arbitrary velocity $\beta_{0}$,

$$
\begin{aligned}
& \frac{d}{d s} D_{1}=D_{2}+H D_{3}, \\
& \frac{d}{d s} D_{2}=\left[D_{4}-H D_{1}\right] H-\left[K_{x}^{2}+g\right] D_{1}+N D_{3}-\left[F_{1} D_{1}+F D_{3}\right] \delta\left(s-s_{I P}\right)+K_{x}, \\
& \frac{d}{d s} D_{3}=D_{4}-H D_{1}, \\
& \frac{d}{d s} D_{4}=-\left[D_{2}+H D_{3}\right] H+N D_{1}-\left[K_{y}^{2}-g\right] D_{3}-\left[F_{1} D_{3}+F D_{1}\right] \delta\left(s-s_{I P}\right)+K_{y},
\end{aligned}
$$

the new Hamiltonian reads

$$
\begin{aligned}
\tilde{\mathcal{H}}= & \frac{1}{2}\left\{\left[\tilde{p}_{x}+\tilde{y} H\right]^{2}+\left[\tilde{p}_{y}-\tilde{x} H\right]^{2}\right\}+\frac{1}{2}\left\{\left[K_{x}^{2}+g\right] \tilde{x}^{2}+\left[K_{y}^{2}-g\right] \tilde{y}^{2}\right\}-N \tilde{x} \tilde{y} \\
& -\frac{1}{2}\left[\left(K_{x} D_{1}+K_{y} D_{3}\right)-1 / \gamma_{0}^{2}\right] \tilde{p}_{z}^{2}-\frac{1}{\beta_{0}^{2}} h \frac{2 \pi}{L} \frac{e V}{E_{0}} \cos \varphi_{\mathrm{rf}} \frac{1}{2}\left\{\tilde{z}+\left[\tilde{p}_{x} D_{1}-\tilde{x} D_{2}+\tilde{p}_{y} D_{3}-\tilde{y} D_{4}\right]\right\}^{2} \\
& +\left[\frac{1}{2} \tilde{x}^{2} F_{1}+\frac{1}{2} \tilde{y}^{2} F_{2}+F x y\right] \delta\left(s-s_{\mathrm{IP}}\right) .
\end{aligned}
$$

Note that the dispersion vector $\vec{D}$ is the periodic solution of the linearized equations of orbital motion when the cavities are excluded and $p_{z}=1$.

The coupling terms [see (D17)] arising from the orbit curvature disappear. Instead, there is a term

$$
\begin{aligned}
& -\frac{1}{2}\left\{\tilde{z}+\left[\tilde{p}_{x} D_{1}-\tilde{x} D_{2}+\tilde{p}_{y} D_{3}-\tilde{y} D_{4}\right]\right\}^{2} \\
& \times \frac{1}{\beta_{0}^{2}} h \frac{2 \pi}{L} \frac{e V}{E_{0}} \cos \varphi_{\mathrm{rf}}
\end{aligned}
$$

which disappears when all four dispersion terms $\left(D_{1}, D_{2}, D_{3}, D_{4}\right)$ are equal to zero at the location of the cavities.

For further analysis we split the Hamiltonian (D17) into three parts,

$$
\tilde{\mathcal{H}}=\tilde{\mathcal{H}}^{0}+\tilde{\mathcal{H}}^{1}+\tilde{\mathcal{H}}^{2}
$$

with 


$$
\begin{aligned}
\tilde{\mathcal{H}}^{0}= & \frac{1}{2}\left\{\left[\tilde{p}_{x}+\tilde{y} H\right]^{2}+\left[\tilde{p}_{y}-\tilde{x} H\right]^{2}\right\}+\frac{1}{2}\left\{\left[K_{x}^{2}+g\right] \tilde{x}^{2}+\left[K_{y}^{2}-g\right] \tilde{y}^{2}-N \tilde{x} \tilde{y}\right\} \\
& -\frac{1}{2}\left[\left(K_{x} D_{1}+K_{y} D_{3}\right)-1 / \gamma_{0}^{2}\right] \tilde{p}_{z}^{2}-\frac{1}{\beta_{0}^{2}} h \frac{2 \pi}{L} \frac{e V}{E_{0}} \cos \varphi_{\mathrm{rf}} \frac{1}{2} \tilde{z}^{2}, \\
\tilde{\mathcal{H}}^{1}= & -\frac{1}{\beta_{0}^{2}} h \frac{2 \pi}{L} \frac{e V}{E_{0}} \cos \varphi_{\mathrm{rf}} \frac{1}{2}\left[\tilde{p}_{x} D_{1}-\tilde{x} D_{2}+\tilde{p}_{y} D_{3}-\tilde{y} D_{4}\right]\left\{2 \tilde{z}+\left[\tilde{p}_{x} D_{1}-\tilde{x} D_{2}+\tilde{p}_{y} D_{3}-\tilde{y} D_{4}\right]\right\}, \\
\tilde{\mathcal{H}}^{2}= & {\left[\frac{1}{2} \tilde{x}^{2} F_{1}+\frac{1}{2} \tilde{y}^{2} F_{2}+F x y\right] \delta\left(s-s_{\mathrm{IP}}\right), }
\end{aligned}
$$

where we have gathered in $\tilde{\mathcal{H}}^{1}$ all terms of the cavities producing synchrobetatron coupling and in $\tilde{\mathcal{H}}^{2}$ the terms resulting from the beam-beam interaction.

In terms of the variables $\tilde{x}, \tilde{p}_{x}, \tilde{y}, \tilde{p}_{y}, \tilde{z}, \tilde{p}_{z}$, Eq. (D4) then takes the form

$$
\frac{d}{d s} \overrightarrow{\tilde{x}}=\underline{A}^{(0)} \overrightarrow{\tilde{x}}+\underline{A}^{(1)} \overrightarrow{\tilde{x}}+\underline{A}^{(2)} \overrightarrow{\tilde{x}}
$$

with

$$
\begin{aligned}
\underline{A}^{(1)}(s) & =\frac{1}{\beta_{0}^{2}} \frac{e V(s)}{E_{0}} h \frac{2 \pi}{L} \cos \varphi_{\mathrm{rf}}\left(\begin{array}{ccccccc}
D_{2} \vec{D} & -D_{1} \vec{D} & D_{4} \vec{D} & -D_{3} \vec{D} & -\vec{D} & \overrightarrow{0}_{4} \\
0 & 0 & 0 & 0 & 0 & 0 \\
-D_{2} & D_{1} & -D_{4} & D_{3} & 0 & 0
\end{array}\right), \\
\underline{A}^{(2)}(s) & =\left(\begin{array}{cccccc}
0 & 0 & 0 & 0 & 0 & 0 \\
-F_{1} & 0 & -F & 0 & 0 & 0 \\
0 & 0 & 0 & 0 & 0 & 0 \\
-F & 0 & -F_{2} & 0 & 0 & 0 \\
0 & 0 & 0 & 0 & 0 & 0 \\
0 & 0 & 0 & 0 & 0 & 0
\end{array}\right) \delta\left(s-s_{\mathrm{IP}}\right),
\end{aligned}
$$

$$
\begin{aligned}
& \underline{A}^{(0)} \overrightarrow{\tilde{x}}=-\underline{J} \frac{\partial \tilde{\mathcal{H}}^{0}}{\partial \overrightarrow{\tilde{x}}} \Longrightarrow A_{m n}^{(0)}=-S_{m l} \frac{\partial^{2} \tilde{\mathcal{H}}^{0}}{\partial y_{l} \partial y_{n}}, \\
& \underline{A}^{(1)} \overrightarrow{\tilde{x}}=-\underline{J} \frac{\partial \tilde{\mathcal{H}}^{1}}{\partial \overrightarrow{\tilde{x}}} \Longrightarrow A_{m n}^{(1)}=-S_{m l} \frac{\partial^{2} \tilde{\mathcal{H}}^{1}}{\partial y_{l} \partial y_{n}}, \\
& \underline{A}^{(2)} \overrightarrow{\tilde{x}}=-\underline{J} \frac{\partial \tilde{\mathcal{H}}^{2}}{\partial \overrightarrow{\tilde{x}}} \Longrightarrow A_{m n}^{(2)}=-S_{m l} \frac{\partial^{2} \tilde{\mathcal{H}}^{2}}{\partial y_{l} \partial y_{n}}
\end{aligned}
$$

In detail, one obtains from Eqs. (D19)

$$
\underline{A}^{(0)}(s)=\left(\begin{array}{cc}
\underline{A}_{4 \times 4}^{(\beta)}(s) & \underline{0}_{2 \times 2} \\
\underline{0}_{2 \times 4} & \underline{A}_{2 \times 2}^{(z)}(s)
\end{array}\right),
$$

with $\underline{A}_{4 \times 4}^{(\beta)}(s)$ and $\underline{A}_{2 \times 2}^{(z)}(s)$

$$
\begin{gathered}
\left(\begin{array}{cccc}
0 & 1 & H & 0 \\
-\left(K_{x}^{2}+g+H^{2}\right) & 0 & N & H \\
-H & 0 & 0 & 1 \\
N & -H & -\left(K_{y}^{2}-g+H^{2}\right) & 0
\end{array}\right), \\
\left(\begin{array}{ccc}
\text { (D224) } \\
0 \\
\frac{1}{\beta_{0}^{2}} \frac{e V(s)}{E_{0}} h \frac{2 \pi}{L} \cos \varphi & -\left[\left(K_{x} D_{1}+K_{y} D_{3}\right)-1 / \gamma_{0}^{2}\right]
\end{array}\right),
\end{gathered}
$$

\section{B. The eigenvalue spectrum of the orbital revolution matrix}

The solution of the original Hamiltonian (D1) can be written as

$$
\vec{x}(s)=\underline{M}\left(s, s_{0}\right) \vec{x}\left(s_{0}\right),
$$

which defines the linear transfer matrix $\underline{M}\left(s, s_{0}\right)$ corresponding to the variables $\left(x, y, z ; p_{x}, p_{y}, p_{z}\right)$.

Note that $\underline{M}\left(s, s_{0}\right)$ is symplectic [18]

$$
\underline{M}^{T}\left(s, s_{0}\right) \underline{J} \underline{M}\left(s, s_{0}\right)=\underline{J} .
$$

Thus the (normalized) eigenvectors of the revolution matrix

$$
\begin{gathered}
\underline{M}\left(s_{0}+L, s_{0}\right) \overrightarrow{\boldsymbol{v}}_{k}\left(s_{0}\right)=e^{-i 2 \pi Q_{k}} \overrightarrow{\boldsymbol{v}}_{k}\left(s_{0}\right), \\
Q_{-k}=-Q_{k}, \quad(k=I, I I, I I I)
\end{gathered}
$$

coupling induced by the cavities and $\underline{A}^{(2)}$ results from the beam-beam kick.

The tune shifts induced by $\underline{A}^{(1)}$ and $\underline{A}^{(2)}$ can be obtained by determining the tunes with and without $\tilde{\mathcal{H}}^{1}$ and $\tilde{\mathcal{H}}^{2}$ or by perturbative methods as described in Refs. [18,19]. In a similar way one can calculate the distortion of the dispersion induced by the beam-beam kick.
${ }^{4}$ Neglecting all kinds of coupling, such as synchrobetatron coupling $\left[\underline{A}^{1}(s)=0\right]$ and coupling of betatron motion $(N=$ $H=0$, no skew quadrupoles and no solenoids), we obtain the dispersion formalism used in Ref. [1]. 
obey the orthogonality relations [see Eq. (A4)], where we have assumed that the stability condition

$$
Q_{k} \text { real number }
$$

is satisfied.

Putting

$$
\overrightarrow{\boldsymbol{v}}_{k}(s)=\overrightarrow{\hat{\boldsymbol{v}}}_{k}(s) e^{-i 2 \pi Q_{k}(s / L)},
$$

we obtain from (D28)

$$
\overrightarrow{\hat{v}}_{k}(s+L)=\overrightarrow{\hat{v}}_{k}(s)
$$

(Floquet theorem). Using this result, action-angle variables for coupled motion can be introduced as described in Ref. [18].

The introduction of the dispersion has been accomplished by the matrix $\underline{K}$ [see Eq. (D12)]

$$
\overrightarrow{\tilde{x}}=\underline{K} \vec{x}, \quad \vec{x}=\underline{K}^{-1} \overrightarrow{\tilde{x}} .
$$

Using the transfer matrix $\underline{M}\left(s, s_{0}\right)$ we can write

$$
\overrightarrow{\tilde{x}}(s)=\underline{K}(s) \underline{M}\left(s, s_{0}\right) \underline{K}^{-1}\left(s_{0}\right) \overrightarrow{\tilde{x}}\left(s_{0}\right),
$$

showing that the transfer matrix $\underline{\tilde{M}}\left(s, s_{0}\right)$ can be represented as [20]

$$
\underline{\tilde{M}}\left(s, s_{0}\right)=\underline{K}(s) \underline{M}\left(s, s_{0}\right) \underline{K}^{-1}\left(s_{0}\right) .
$$

In particular, one has

$$
\underline{\tilde{M}}\left(s_{0}+L, s_{0}\right)=\underline{K}\left(s_{0}\right) \underline{M}\left(s_{0}+L, s_{0}\right) \underline{K}^{-1}\left(s_{0}\right) .
$$

For the eigenvectors one gets

$$
\overrightarrow{\tilde{v}}_{k}\left(s_{0}\right)=\underline{K}\left(s_{0}\right) \overrightarrow{\boldsymbol{v}}_{k}\left(s_{0}\right)
$$

since

$$
\begin{aligned}
\underline{\tilde{M}}\left(s_{0}+L, s_{0}\right) \underline{K}\left(s_{0}\right) \overrightarrow{\boldsymbol{v}}_{k}\left(s_{0}\right)= & \underline{K}\left(s_{0}\right) \underline{M}\left(s_{0}+L, s_{0}\right) \\
& \times \underline{K}^{-1}\left(s_{0}\right) \underline{K}\left(s_{0}\right) \overrightarrow{\boldsymbol{v}}_{k}\left(s_{0}\right) \\
= & \underline{K}\left(s_{0}\right) \underline{M}\left(s_{0}+L, s_{0}\right) \overrightarrow{\boldsymbol{v}}_{k}\left(s_{0}\right) \\
= & e^{-i 2 \pi Q_{k}} \underline{K}\left(s_{0}\right) \overrightarrow{\boldsymbol{v}}_{k}\left(s_{0}\right)
\end{aligned}
$$

Note that the eigenvalues and thus the $Q$ values remain unchanged;

$$
\tilde{Q}_{k}=Q_{k} .
$$

The orthogonality relations for the new eigenvectors $\overrightarrow{\tilde{v}}_{k}$ are still valid. Furthermore, $\underline{\tilde{M}}\left(s, s_{0}\right)$ is symplectic as it is obtained by a similarity transformation of $\underline{M}$ with $\underline{K}$.

\section{Decoupled motion; Twiss parameters}

In the case of vanishing dispersion within the cavities, the revolution matrix $\underline{\tilde{M}}\left(s_{0}+L, s_{0}\right)$ has the simple blockdiagonal form

$$
\underline{\tilde{M}}\left(s_{0}+L, s_{0}\right)=\left(\begin{array}{cc}
\underline{M}_{(4 \times 4)}^{(\beta)}\left(s_{0}+L, s_{0}\right) & \underline{0}_{(4 \times 2)} \\
\underline{0}_{(2 \times 4)}^{(z)} & \underline{M}_{(2 \times 2)}^{\left(s_{0}+L, s_{0}\right)}
\end{array}\right),
$$

where $\underline{M}_{(4 \times 4)}^{(\beta)}\left(s_{0}+L, s_{0}\right)$ corresponds to the (transverse) betatron motion and $\underline{M}_{(2 \times 2)}^{(z)}\left(s_{0}+L, s_{0}\right)$ to the (longitudinal) synchrotron oscillations.

Furthermore, the two-dimensional revolution matrix $\underline{M}_{(2 \times 2)}^{(z)}\left(s_{0}+L, s_{0}\right)$, which is defined by the equations of synchrotron motion

$$
\begin{aligned}
\frac{d}{d s} \tilde{z} & =-\left[\left(K_{x} D_{x}+K_{y} D_{y}\right)-1 / \gamma_{0}^{2}\right] \tilde{p}_{z}, \\
\frac{d}{d s} \tilde{p}_{z} & =\frac{1}{\beta_{0}^{2}} h \frac{2 \pi}{L} \frac{e V\left(s_{0}\right)}{E_{0}} \cos \varphi_{\mathrm{rf}} \tilde{z}
\end{aligned}
$$

[see Eq. (D25)], can be represented in the form

$$
\underline{M}_{(2 \times 2)}^{(z)}\left(s_{0}+L, s_{0}\right)=\left(\begin{array}{cc}
\cos 2 \pi Q_{z}+\alpha_{z} \sin 2 \pi Q_{z} & \beta_{z} \sin 2 \pi Q_{z} \\
-\gamma_{z} \sin 2 \pi Q_{z} & \cos 2 \pi Q_{z}+\alpha_{z} \sin 2 \pi Q_{z}
\end{array}\right)
$$

with

$$
\beta_{z} \gamma_{z}=\alpha_{z}^{2}+1
$$

$$
\begin{aligned}
& \overrightarrow{\tilde{v}}_{k}=\left(\begin{array}{c}
\vec{v}_{k}^{(\beta)} \\
\overrightarrow{0}_{2}
\end{array}\right), \quad(k=I, I I), \\
& \overrightarrow{\tilde{v}}_{I I I}=\left(\begin{array}{c}
\overrightarrow{0}_{4} \\
\vec{t}_{z}
\end{array}\right),
\end{aligned}
$$

$$
\alpha_{z}=\alpha_{z}\left(s_{0}\right), \quad \beta_{z}=\beta_{z}\left(s_{0}\right), \quad \gamma_{z}=\gamma_{z}\left(s_{0}\right) .
$$

From these equations one sees that the eigenvectors $\overrightarrow{\tilde{v}}_{k}\left(s_{0}\right)$ can be written as
In the absence of skew quadrupoles $(N=0)$ and solenoids $(H=0)$ [the coupling term $F x y$ vanishes in (D19)], the betatron oscillations are decoupled, leading to 


$$
\begin{gathered}
\underline{M}_{(4 \times 4)}^{(\beta)}\left(s_{0}+L, s_{0}\right)=\left(\begin{array}{cc}
\underline{M}_{(2 \times 2)}^{(x)}\left(s_{0}+L, s_{0}\right) & \underline{0}_{(2 \times 2)} \\
\underline{0}_{(2 \times 2)} & \underline{M}_{(2 \times 2)}^{(w)}\left(s_{0}+L, s_{0}\right)
\end{array}\right), \\
\underline{M}_{(2 \times 2)}^{(y)}\left(s_{0}+L, s_{0}\right)=\left(\begin{array}{cc}
\cos 2 \pi Q_{w}+\alpha_{w} \sin 2 \pi Q_{w} & \beta_{w} \sin 2 \pi Q_{w} \\
-\gamma_{w} \sin 2 \pi Q_{w} & \cos 2 \pi Q_{w}+\alpha_{w} \sin 2 \pi Q_{w}
\end{array}\right), \\
\beta_{w} \gamma_{w}=\alpha_{w}^{2}+1, \quad(w \equiv x, y) .
\end{gathered}
$$

As a result, the vectors $\overrightarrow{\tilde{v}}_{I}$ and $\overrightarrow{\tilde{v}}_{I I}$ then take a form similar to $\overrightarrow{\tilde{v}}_{I I I}$,

$$
\vec{v}_{I}^{(\beta)}=\left(\begin{array}{c}
\vec{t}_{x} \\
\overrightarrow{0}_{2}
\end{array}\right), \quad \vec{v}_{I I}^{(\beta)}=\left(\begin{array}{c}
\overrightarrow{0}_{2} \\
\vec{t}_{y}
\end{array}\right), \quad \vec{t}_{w} \equiv\left(\begin{array}{c}
t_{w 1} \\
t_{w 2}
\end{array}\right)=\frac{1}{\sqrt{2 \beta_{w}\left(s_{0}\right)}}\left(\begin{array}{c}
\beta_{w}\left(s_{0}\right) \\
-\left[\alpha_{w}\left(s_{0}\right)+i\right]
\end{array}\right) e^{-i \psi_{w}\left(s_{0}\right)}, \quad(w \equiv x, y) .
$$

It is easy to generalize this treatment to the coupled case; see Ref. [18].

[1] K. Hirata, H. Moshammer, and F. Ruggiero, KEK Report No. 92-117, 1992.

[2] K. Hirata, SLAC Report No. SLAC-PUB-6375,1994.

[3] H. Grote and F. C. Iselin, Report No. CERN/SL/90-13(AP) (rev.4), 1995.

[4] F. Schmidt, Report No. CERN/SL/90-11(AP), 1990; G. Ripken and F. Schmidt, Report No. CERN/SL/95-12(AP), 1995; DESY Report No. 95-063, 1995.

[5] D. P. Barber, K. Heinemann, H. Mais, and G. Ripken, DESY Report No. 91-146, 1991.

[6] I. Borchardt, E. Karantzoulis, H. Mais, and G. Ripken, Z. Phys. C 39, 339 (1988).

[7] I. Borchardt, E. Karantzoulis, H. Mais, and G. Ripken, Z. Phys. C 41, 25 (1988).

[8] E. Forest and K. Ohmi, KEK Report No. 92-14, 1992.

[9] D. P. Barber, K. Heinemann, G. Ripken, and F. Schmidt, DESY Report No. 96-156, 1996.
[10] K. Heinemann, G. Ripken, and F. Schmidt, DESY Report No. 95-189, 1995.

[11] W. Gröbner, Differentialgleichungen, Erster Teil; Gewöhnliche Differentialgleichungen (BI-Wissenschaftsverlag, Mannheim, Wien, Zürich, 1977).

[12] H. Mais and C. Mari, Report No. CERN-94-01, 1994, p. 499.

[13] G. Ripken, DESY Report No. 85-84, 1985.

[14] D. P. Barber, G. Ripken, and F. Schmidt, DESY Report No. 87-36, 1987.

[15] C. J. A. Corsten and H. J. Hagedoorn, Nucl. Instrum. Methods Phys. Res. 212, 37 (1983).

[16] H. Mais and G. Ripken, DESY Report No. 86-29, 1986.

[17] G. Ripken and E. Karantzoulis, DESY Report No. 86-29, 1986.

[18] F. Willeke and G. Ripken, DESY Report No. 88-114, 1988; F. Willeke and G. Ripken, DESY Report No. 90-001, 1990.

[19] D. P. Barber, K. Heinemann, G. Ripken, and F. Willeke, Report No. DESY HERA 94-02, 1994.

[20] D. P. Barber and G. Ripken, in Handbook of Accelerator Physics and Engineering, edited by A. W. Chao and M. Tigner (World Scientific, Singapore, 1999). 